\title{
Continuous single cell transcriptome dynamics reveal a default vascular smooth muscle fate of FLK1 mesoderm
}

Haiyong Zhao ${ }^{1}$ and Kyunghee Choi ${ }^{1,2, *}$

${ }^{1}$ Department of Pathology and Immunology, Washington University School of Medicine, St. Louis, MO, USA

${ }^{2}$ Graduate School of Biotechnology, Kyung Hee University, Yong In, The Republic of Korea

${ }^{*}$ Corresponding author, kchoi@wustl.edu

Running title: Single cell transcriptome of Flk1-mesoderm

Keywords: Single cell RNA sequencing, Flk1/Kdr, Etv2, hemangiogenesis, vascular smooth muscle, mouse embryonic stem, yolk sac 


\section{Abstract}

Blood and endothelial cells arise from hemangiogenic progenitors that are specified from FLK1expressing mesoderm by the transcription factor ETV2. FLK1 mesoderm also contributes to other tissues, including vascular smooth muscle (VSM) and cardiomyocytes. However, the developmental process of FLK1 mesoderm generation and its allocation to various cell fates remain obscure. Recent single cell RNA-sequencing (scRNA-seq) studies of early stages of embryos, or in vitro differentiated human embryonic stem (ES) cells have provided unprecedented information on the spatiotemporal resolution of cells in embryogenesis. These snapshots nonetheless offer insufficient information on dynamic developmental processes due to inadvertently missing intermediate states and unavoidable batch effects. Here we performed scRNA-seq of in vitro differentiated ES cells as well as extraembryonic yolk sac cells, which contain the very first arising hemangiogenic and VSM lineages, to capture the continuous developmental process leading to hemangiogenesis. We found that hemangiogenic progenitors from ES cells develop through intermediate gastrulation stages, which were gradually specified by 'relay'-like highly overlapping transcription factor modules. Unexpectedly, VSM and hemangiogenic lineages share the closest transcriptional program. Moreover, transcriptional program of the Flk1 mesoderm was maintained in the VSM lineage, suggesting the VSM lineage may be the default pathway of FLK1 mesoderm. We also identified cell adhesion signals possibly contributing to ETV2-mediated activation of the hemangiogenic program. This continuous transcriptome map will facilitate both basic and applied studies of mesoderm and its derivatives. 


\section{Introduction}

Previous studies have established that blood and endothelial cells differentiate from FLK1expressing mesoderm ${ }^{1}$. FLK1 mesoderm can also contribute to vascular smooth muscle (VSM), cardiac lineages, and paraxial mesoderm tissues ${ }^{2,3}$. However, high plasticity, heterogeneity, and rapid differentiation of early embryo cells have hampered investigations on the lineage relationships among these cell types, the mechanisms underlying the lineage restriction steps from pluripotent cells to FLK1 mesoderm, and the following commitment to different downstream lineages. Recent progress in scRNA-seq technology has unprecedentedly advanced our understanding of the complex cell constitution at discrete time points ${ }^{4-7}$ or in sorted cell populations $s^{8,9}$ in early embryo development or in vitro differentiated ES cells ${ }^{10,11}$. However, none of these studies have provided a complete process from pluripotent cells to FLK1 mesoderm due to missing intermediate states or batch effects. Therefore, there is still a gap in our current knowledge on the continuous sequence of FLK1 mesoderm development and its segregation into the hemangiogenic lineage.

Mouse ES cells in culture differentiate in an asynchronous manner giving rise to embryoid bodies (EB). Both undifferentiated pluripotent cells and differentiated progenies ${ }^{12,13}$ coexist in mouse EBs of early stages of differentiation. We reasoned that cells in the intermediate states also exist in the same culture, representing the full spectrum of differentiation, which would allow a unique opportunity to delineate a continuous differentiation process without batch effects. By taking advantage of the asynchronous nature of the differentiation of mouse ES cells and the culture conditions that favor hemangiogenic lineage differentiation, we performed scRNA-seq of EB cells to elucidate a continuous developmental path from the pluripotent state to FLK1 mesoderm, and to the hemangiogenic lineage. We found that the cardiac lineage branched out immediately after nascent mesoderm specification. Intermediate states positioned in-between ES and FLK1 mesoderm were distinguished by a 
few temporally overlapping transcription factor modules, suggesting a plastic and combinational regulatory mechanism in Flk1 mesoderm development. Notably, expression of most of the characteristic genes of Flk1 mesoderm continuously increased in the VSM branch, implying a 'default' route. Our data suggested that ETV2 turns on the hemangiogenic program from FLK1 mesoderm by restraining the VSM fate. We further identified cell adhesion-SRC kinase signaling as a potential regulator of ETV2-mediated hemangiogenic lineage specification. These studies provide a comprehensive description of mesoderm and hematopoietic/endothelial cell lineage development at the single cell level.

\section{Results}

\section{EBs contain undifferentiated pluripotent cells and FLK1 mesoderm tissues}

We performed scRNA-seq using day 4 EB cells in hemangiogenic differentiation. After filtering out low-quality cells, 1848 cells were kept for further analysis, which had 4373 genes/27272 unique mRNAs detected per cell in average (Figure 1A). Day 4 EB cells were grouped into 18 populations (Figure 1B, supplemental Figure 1A). 936 genes were differentially upregulated across the populations (supplemental Table 1). t-Stochastic Neighbor Embedding (t-SNE) indicated that most clusters did not form a distinct, wellsegregated population (Figure 1B). Instead, distinct populations branched out gradually from the trunk, underpinning continuous developmental processes. We assigned different fates to the branches according to the expression of related marker genes (supplemental Table 1). SPRING ${ }^{14}$, a different tool for high dimension scRNA-seq data visualization, produced a similar cell clustering pattern (supplemental Figure 1B). High expression of Sox2, Pou5f1/Oct4, Nanog and Zfp42/Rex1 highlights the maintenance of undifferentiated naïve pluripotent cells in the EBs (Figure 1C, 1D). FLK1 mesoderm and the hemangiogenic lineage, as marked by high expression of $F / k 1 / K d r$ and Etv2, and upregulation of the ETV2 target genes Tal1 and Gata2, clustered at the other end of the ES cell population (Figure 1C, 
1D). Expression of related genes showed a gradual shift in intermediate clusters from the pluripotent ES population to the Flk1 mesoderm branch, revealing continuous intermediate states from ES to FLK1 mesoderm that are accessible in EBs.

\section{scRNA-seq captured multiple intermediate stages from naïve pluripotency to FLK1 derivatives in EBs}

To further determine developmental relationships among the clusters in-between the ES and Flk1 hemangiogenic populations, we first examined the dynamics of the loss of pluripotency and the bifurcation of mesoderm and endoderm fates. Pluripotency is maintained in two states: the 'naïve' and the 'primed' ${ }^{15}$. The former represents the primitive phase and highly expresses Zfp42/Rex1; while the latter loses Zfp42 expression and harbors an unstable pluripotency gene network, which marks a nascent stage of gastrulation/mesendoderm ${ }^{15}$. We picked out clusters which are located in the endoderm path $(4,5,8,9,11$ and 17 in Figure 1B), and re-clustered the cells based on 163 most differentially expressed genes among these cells (Figure 2A). A narrow "path" from Utf1-expressing pluripotent population leads to the Sox17-expressing endoderm (red dash line-surrounded region), next to the sprouting Mesp1-expressing nascent mesoderm. We next ordered cells in the endoderm path into a pseudo-time developmental line and examined the kinetics of 163 most differentially expressed genes (Figure 2B-2D, supplemental Figure 2C). At the beginning of the path, we observed an exit from the naïve pluripotency (Figure 2C, flanking the blue dash line). Unexpectedly, expression of a small group of genes (represented by Dppa3, brown box in Figure 2B, and in Figure 2C) already displayed strong heterogeneity in the Zfp42 -expressing population. Gradual loss of the expression of these genes preceded the exit from naïve pluripotency. This may suggest an extra layer of guarding mechanisms of pluripotency beyond the naïve circuit (as represented by the genes in the blue box in Figure 2B, which have relatively constant expression in the naïve pluripotency stage). Primitive streak markers 
such as $T$ and Fgf5 were up regulated in cells exiting from the naïve pluripotent state and entering the mesendoderm stage (Figure 2A). Shortly after, the endoderm and mesoderm lineages bifurcated. Fgf5 expression became more enriched in endoderm, while T expression was exclusively enriched in mesoderm (Figure 2A). Gata6 and Gsc were expressed in both mesoderm and endoderm, although they were expressed more extensively in the latter (Figure 2A). The majority of cells in the endoderm population were separated from the trunk, with very few Foxa2-high expressing cells in the intermediate state. When we lowered down the stringency and included more variable genes to cluster the cells, the connection between endoderm and the mesendoderm cluster becomes more obvious (supplemental Figure 2AB). These results might reflect that the core endoderm genes form a strong self-enforcing circuit, rendering the intermediate specification state relatively unstable.

The immediate upstream population of $F / k 1^{+}$mesoderm expressed Pdgfra and Mesp1 (Figure 2E, blue dash line-marked region). These cells also expressed low levels of Tbx6, implying that they are to some extent 'primed' for paraxial mesoderm, which generates tissues like skeletal muscle and cartilage. As such, they might represent an early ancestral state for both FLK1 lateral plate mesoderm and paraxial mesoderm. Consistently, cells in this region have an expression pattern similar to the posterior primitive streak of mouse embryo at E7.0, where nascent mesoderm emerges (Figure 2F). Furthermore, these cells were transcriptionally similar to Mesp1-labeled early mesoderm cell population in mouse E6.75 embryos in Lescroart et al's recent scRNA-seq data ${ }^{8}$. This embryonic population of cells still expressed high level of the pluripotency marker Pou5f1, already expressed high levels of Mesp1 and Pdgfra, while expressed low levels of Tbx6 and has not upregulated Kdr yet (cluster 1 in supplemental Figure 2D). The cardiac lineage branched out mainly from the Pdgfr $\alpha^{+}$Flk1- early mesoderm population, while a small group of Flk1 mesoderm also expressed the cardiac transcription factor Is/1 (Figure 2G). Notably, VSM and hemangiogenic 
lineages directly bifurcated from a Flk1-expressing population, suggesting a common origination (Figure $2 \mathrm{H}$ ). RNA velocity estimation of the variable genes, based on the abundance of unspliced and spliced $m \mathrm{RNAs}^{16}$, revealed persistent overall cell state dynamics from naive pluripotency to differentiation branches (supplemental Figure 2E). These results suggested a continuous process from pluripotency to Flk1 mesoderm and its derivate tissues (Figure 2I).

\section{Cells corresponding to different development stages proliferate equally}

It was possible that cells in alternative differentiation paths might have different proliferation rate. The expression pattern of typical S-phase marker genes showed no obvious bias in different cell populations (Figure 3A, supplemental Figure 3A). Using the cell cycle analysis tool in the Seurat package ${ }^{17}$, we assigned each cell to a specific phase in the cell cycle and found that the majority of cells in the mesoderm developmental route proliferated robustly (Figure 3B, supplemental Figure 3B). Analysis of cell proliferation using the violet proliferation dye 450 (VPD450) also confirmed that EB cells with different levels of differentiation (indicated by reporter of the hemangiogenic marker gene Etv2) proliferated similarly in our system (Figure 3C, top). As a control, ERK inhibitor PD0325901-mediated differentiation block and cell cycle delay was observed in the VPD450 dilution assay (Figure $3 C$, bottom).

\section{Transcriptome dynamics in hemangiogenic lineage development}

Next, we retrieved a continuous process of hemangiogenic lineage development by manually choosing the populations corresponding to the shortest path from the naïve pluripotent state to the hemangiogenic branch and reconstructed a fine developmental route (Figure $4 \mathrm{~A}$ ). A group of Flk1-expressing cells achieved the highest level of Etv2 expression, followed by dramatic up regulation of the ETV2 target genes, $\mathrm{Tal}(\mathrm{Scl})$ and Gata2, confirming that the 
threshold-level of Etv2 expression is necessary for hemangiogenic lineage specification ${ }^{18}$ (Figure 4A). We ordered the cells into a 1-D pseudo-time line and assigned the populations to specific stages according to the dynamics of marker gene expression (Figure 4B). 68 most variable transcription regulation-related factors were clustered based on their expression along the pseudo-time line (Figure 4C). They aggregated into a few obvious modules covering different developmental stages (blue dash line boxed regions in the heatmap). Notably, the naïve pluripotency module (box II, Figure 4C) and the hemangiogenic module (box III) were relatively independent, while each of the intermediate gastrulation modules were shared by adjacent stages (box I, IV, and V, Figure 4C). The gene network constructed based on the gene co-expression also highlighted the collaboration among transcription factors of individual modules (Figure 4D). This stepwise combinatorial usage of different regulatory circuits fits the diverse lineage specifications in gastrulation. Consistently, the intermediate populations were less committed, largely interchangeable ${ }^{19}$, and more similar to each other in transcriptome (Figure 4E, supplemental Figure 4A, 4B). These results suggested that the intermediate states constitute a 'relay'-like multistep specification process (Figure 4F). Exit from the naive pluripotency and activation of the hemangiogenic program might be the two critical transitions in hemangiogenic lineage development.

\section{Cell adhesion signaling potentiates ETV2-mediated hemangiogenic fate specification}

Due to intracellular noises and microenvironmental influences, gene expression within individual cells keeps fluctuating. scRNA-seq analysis offers an opportunity to utilize this luxuriant gene expression heterogeneity to assess a regulatory network. We previously reported that Etv2 expression above a threshold-level is essential to activate hemangiogenic genes $^{18}$. We confirmed this at a single cell endogenous mRNA level (Figure 5A). We noticed that the kinetics of upregulation of ETV2 target genes soon became extremely steep after Etv2 expression reached to a specific level, suggesting an Etv2 mediated sensitive "switch" 
mechanism in activating its downstream target genes. We explored potential ETV2 cooperating genes for triggering this sensitive switch by examining potential upstream regulators of Tal1 and Lmo2, two ETV2 direct target genes that are critical for establishing the hemangiogenic lineage ${ }^{20,21}$. First, we assumed that if more gene A-expressing cells simultaneously expressed gene $B$, it is likely that gene A expression depends on gene $B$ (Figure 5B, see methods for more details). From the population where ETV2 starts to activate hemangiogenic genes (population 12 in Figure 1B), we assessed the possibility of a given gene being required for Tal1 or Lmo2's expression (Figure 5C, 5D). Etv2 and Kdr/Flk1 were at the top of the list of genes required for Tal1 or Lmo2 expression (Figure 5C, 5D, supplemental Table 2), consistent with the finding that VEGF-FLK1 signaling ensures Etv2 threshold expression to activate hemangiogenic genes ${ }^{18}$. The comparison of the top 100 genes required for Tal1 expression and the top 100 required for Lmo2 expression generated 66 in common, of which 22 were annotated to cell adhesion/MAPK-related signaling (supplemental Figure 5A, highlighted in supplemental Table 2). The majority of these 22 genes were already highly expressed in populations preceding the hemangiogenic stage (supplemental Figure 5B). To test if cell adhesion signaling plays a role in ETV2-mediated hemangiogenic gene activation, we utilized differentiation of doxycycline (DOX)-inducible Etv2-expressing ES cell line ${ }^{22}$. Since extracellular matrix/cell adhesion signaling converges on the SRC kinase ${ }^{22}$, we added SRC inhibitor PP2 to day 3 EBs, while simultaneously inducing exogenous Etv2 expression. After 24 hours, as expected, DOX-induced Etv2 expression greatly enhanced the FLK1+PDGFR $\alpha^{-}$population that enrich hemangiogenic lineage cells ${ }^{22}$, while inhibition of SRC using PP2 was sufficient to reduce Etv2 overexpression-induced hemangiogenic lineage skewing (supplemental Figure 5C). Given that cell adhesion is necessary for gastrulation as well ${ }^{23}$, it was possible that PP2 affected earlier stages rather than directly affecting ETV2's function. To minimize this possibility, we induced ETV2-mCherry fusion protein expression ectopically in undifferentiated ES cells, 
where endogenous Etv2 level is ignorable ${ }^{18}$ and exogenous Etv2 can induce Flk1 expression. Flk1 is also a target of ETV2 and reciprocal activation between ETV2 and FLK1 signaling is an important mechanism for hemangiogenic fate determination ${ }^{18}$. Exogenous ETV2 induced more than $8 \%$ of FLK1-positive cells in undifferentiated ES cells, while this ratio was significantly reduced by PP2 treatment (Figure 5E). Unexpectedly, PP2 treatment also dramatically reduced ETV2 protein level, with only a limited number of cells achieving threshold expression of Etv2 (Figure 5E). To exclude the possibilities that PP2-mediated SRC inhibition affected DOX-induction system or general gene expression processes, we tested DOX-induced expression of another transcription factor, EOMES, and the constitutively active promoter EF1 $\alpha$-driven expression of the red fluorescent protein mCherry. We found that DOX-induced EOMES protein level only mildly decreased in the presence of PP2, while EF1 $\alpha$-driven mCherry expression was insensitive to this treatment (supplemental Figure 5D). These results suggest that cell adhesion signaling contributes to ETV2-mediated hemangiogenic fate specification via regulation of ETV2 protein production.

\section{FLK1 mesoderm is fated to the vascular smooth muscle (VSM) lineage, while ETV2 skews it into the hemangiogenic fate}

Based on the observation of direct bifurcations of VSM and hemangiogenic lineages from FLK1 mesoderm (Figure 2I), we analyzed the cell populations corresponding to the developmental route of the VSM lineage (Figure 6A, supplemental Figure 6A), and examined expression of the 427 most varied genes along the pseudo-time of differentiation (Figure 6B). Many of the variable genes aggregated as modules. The modules corresponding to mesendoderm, nascent mesoderm, and Flk1-expressing mesoderm stages displayed largely overlapping 'relay'-like patterns. Three groups of genes were most extensively expressed in the VSM branch (Figure 6B, gene clusters a, b and $\mathrm{c}$ in the black dash line boxed region). Of 
the three groups of genes, group b and $\mathrm{c}$ were already up regulated prominently in FLK1 mesoderm, with the cluster c being up regulated even earlier from the mesendoderm stage. The transcription factors Hand1, Tbx20, and Foxf1 were among the gene cluster b (Figure 6B, 6C). Hand1 and Foxf1 have been reported to be important for VSM development ${ }^{24,25}$. Expression of the genes in clusters $b$ and $c$ remained similar in the hemangiogenic branch. Only a small cluster of genes was exclusively up regulated after cells entered the VSM branch (gene cluster a). Importantly, all the VSM transcription factor genes, except Tbx2, were already extensively expressed in the FLK1 mesoderm and constituted a major characteristic of the FLK1 mesoderm (supplemental Figure 6B, in the black dashed box). These results suggest that VSM lineage might be the default fate of the FLK1 mesoderm.

We zoomed into the populations 0, 3, 10, 12 and 13 (as labeled in Figure 1B), corresponding to the bifurcation of hemangiogenic and VSM fates (Figure 6D). Expression of Bmp4, which can enhance VSM development ${ }^{26}$, was dramatically up regulated in the VSM branch (from population 0 (black) to population 13 (orange)), but not in population 12 (green), where Etv2 initiates specification of the hemangiogenic fate (Figure 6D). Hand1 and Foxf1 were dramatically down regulated in population 12 compared to the upstream population 3 , implying an active repression of them by Etv2 (Figure 6E). Consistently, by analyzing Wareing and colleagues' work ${ }^{27}$ we found that re-expression of Etv2 in Etv2-knockout EBs inhibited expression of Hand1 and Foxf1 (Figure 6F). These results suggest that Etv2 represses the default VSM program when specifying the hemangiogenic lineage from FLK1 mesoderm.

\section{FLK1 mesoderm characteristics are enriched in vascular smooth muscle tissue in vivo}

To better understand the VSM program, we further analyzed the overall expression score of the group b genes in Figure 6B (listed in supplemental Table 3). The group b gene score 
reflected the dynamics of FLK1 mesoderm identity in day 4 EB data (supplemental Figure 7A). The cardiac lineage progenitors also had a high group b gene score; as such we termed the group b gene score as the lateral plate mesoderm score, 'LPM-score' (both FLK1 mesoderm and cardiac tissues arise from lateral plate mesoderm). If indeed Flk1 mesoderm default fate is VSM, it was expected that Flk1 mesoderm identities would persist in the VSM lineage. To determine if this is the case in vivo, we performed scRNA-seq of mouse extraembryonic yolk sac, a tissue that generates the first arising hemangiogenic and VSM lineages (Figure 7A). We found that the LPM-score was particularly high in the VSM tissue in mouse yolk sac (Figure 7B), exemplified by the coexpression of the smooth muscle actin gene Acta2 and the transcription factor Foxf1 and Hand1, and the absence of the expression of hematopoietic and endothelial transcription fator $\mathrm{Tal} / \mathrm{Scl}$ (Figure $7 \mathrm{C}$, supplemental Figure 7B). Ibarra-Soria et al. recently reported scRNA-seq of E8.25 mouse embyros ${ }^{5}$. A group of cells in their data, which they assigned as 'amnion', had high LPM-score and expressed VSM markers (Figure 7D-F, supplemental Figure 7C-D). Presumably, this 'amnion' population represents embryonic VSM. Consistently, the 72 marker genes shared by day 4 EB VSM, yolk sac VSM, and 'amnion' were enriched with smooth muscle function annotation (Figure 7G-H). Collectively, the Flk1 mesoderm characteristics persist in embryonic and extraembryonic VSM tissues.

\section{Discussion}

It has been reported that single FLK1 ${ }^{+}$mesoderm cells can form both VSM and endothelial cells in culture ${ }^{2}$. Moreover, sorted $\mathrm{FLK}^{+}$cells are able to form cardiomyocytes and even skeletal muscles ${ }^{19,22}$. However, the lineage relationships among cardiac, vascular smooth muscle, skeletal muscle, and hemangiogenic tissues concerning FLK1 mesoderm allocation remain elusive. Our single cell transcriptome profiling of EB cells revealed a continuous differentiation trajectory. We showed that the cardiac branch mainly segregates out from 
Pdgfr $\alpha^{+} F / k 1^{-}$nascent mesoderm. Unexpectedly, VSM and hemangiogenic lineages have the closest lineage relationship and directly bifurcate from a common group of Flk1-expressing cells. Notably, most FLK1 mesoderm stage markers were further upregulated in the VSM branch, suggesting that VSM might be a default fate of the Flk1-expressing mesoderm. We propose that Etv2 drives the branching of the hemangiogenic lineage from the VSM fate. Expression of cell adhesion signaling coincides with ETV2 target gene activation for hemangiogenic lineage specification. Consistently, inhibition of SRC, a kinase important for cell adhesion signaling, was sufficient to repress hemangiogenic lineage skewing induced by Etv2 overexpression. Meanwhile, critical VSM transcription factors, Hand1 and Foxf1, become actively down regulated. Notably, neither Hand1 nor Foxf1 are direct ETV2 target genes $^{21}$. It will be critical in the future to explore how this repression is achieved.

Hemangiogenic lineage development can be clearly annotated by a series of transcription factor modules, therefore providing strong molecular support for current definition of early embryo developmental stages (Figure 4C). Cells in the gastrulation stages, after exiting pluripotency and before entering the hemangiogenic state, are overall similar to each other in transcriptome, and the underlying transcription factor modules show a 'relay'-like highly overlapping pattern. Consistently, FLK1-PDGFR $\alpha^{-}$, FLK1'PDGFR $\alpha^{+}$and FLK1+PDGFR $\alpha^{+}$ populations in early differentiation of ES cells are reversible/interchangeable ${ }^{19}$. These results suggest that exit from the naïve pluripotency state and activation of the hemangiogenic program might be the two rate-limiting steps in hemangiogenic lineage development, and that the intermediate gastrulating stages are plastic and adapted for stepwise specification to multiple lineage fates through combinational usage of limited regulatory circuits (Figure 7I). 
In summary, our work described a continuous process of a mesoderm lineage tree leading to hemangiogenic and VSM tissue emergence and underlying molecular networks, thereby providing comprehensive information on early embryo and blood/blood vessel development. These findings are fundamental for the study of basic cell fate determination and gene network structures, and for designing more effective strategies to generate hematopoietic and endothelial cells for regenerative medicine.

\section{Methods}

\section{Mouse ES cell culture and differentiation}

Mouse ES cells were maintained and for EB differentiation in serum as previously reported ${ }^{18}$. Briefly, ES cells were maintained on mouse embryo fibroblast (MEF) feeder cell layers in Dulbecco-modified Eagle medium containing 15\% fetal bovine serum, 100 units $/ \mathrm{mL}$ LIF, 1× MEM Non-Essential Amino Acids Solution (Gibco), $1 \times$ Glutamax ${ }^{\mathrm{TM}}$ Supplement (Gibco), and $4.5 \times 10^{-4}$ M 1-Thioglycerol (MTG, Sigma). For feeder-free culture, ES cells were maintained in gelatin-coated dish in Iscove's modified Dulbecco medium (IMDM) with the same supplements as used for maintenance on feeder. For EB differentiation, ES cells were first transferred into feeder-free condition for 2 days, then single-cell suspensions were prepared, and 8,000 cells were added per $\mathrm{mL}$ to a differentiation medium of IMDM containing $15 \%$ differentiation-screened fetal calf serum, $1 \times$ Glutamax, $50 \mu \mathrm{g} / \mathrm{mL}$ ascorbic acid, and $4.5 \times 10^{-4}$ M MTG on a bacteriological Petri dish. The SRC inhibitor PP2 (Sigma) and Doxycycline (Sigma) treatment were performed as indicated in the text.

\section{scRNA-seq}

Yolk sacs were dissected from equal numbers of mouse E9.5 and E10.5 embryos, mixed together and dissociated with $0.25 \%$ collagenase at $37^{\circ} \mathrm{C}$ for 30 minutes. 
Day 4 EBs were dissociated with Accutase solution (Sigma) for 5 minutes into single cells. The single cell suspension was washed with and re-suspended in PBS. Single cell suspension at 300 cells/ $\mu \mathrm{L}$ in PBS were subjected to the Chromium 10X Genomics library construction and HiSeq2500 sequencing (The Genome Technology Access Center, Washington University in St. Louis). The sequenced reads were mapped to the GRCm38 assembly using Cell Range 2.0.1 (10x Genomics).

\section{scRNA-seq data QC}

The scRNA-seq output was imported into Seurat $1.4{ }^{17}$, and genes expressed in at least 3 cells were kept for analysis. Cells with more than $5 \%$ mitochondria reads or less than 2000 unique genes detected were filtered out. 1848 cells were kept for further analysis, which had 4373 genes/27272 unique mRNAs detected per cell in average. Gene expression counts were normalized and log transformed.

\section{scRNA-seq data visualization}

According to the average expression and dispersion gene expression of each gene, we selected 427 most variable genes for further analysis of the remaining cells. nUMls were rescaled to regress the effects of detection depth of each cell and mitochondria reads out. The re-scaled data was run for principle components analysis to reduce dimensions. According to the PCA results the cells were clustered into 18 populations, and also used for t-SNE(tdistributed stochastic neighbor embedding) presentation. Cells in selected populations were imported into Monocle $2^{28}$ for re-clustering, PCA plotting and pseudo-time ordering.

For hierarchical clustering and heatmap plotting of selected genes or cells, the R package 'pheatmap' was used. All scRNA-seq data analyses were finished in R environment. 
For cell cycle score and phase asignment with Seurat, the 'CellCycleScoring' function was invoked.

For LPM-score gene analysis, group b genes in Figure 6B were read and processed by invoking the 'AddModuleScore' function in Seurat.

For SPRING visualization, a matrix of expression counts of the 427 most variable genes vs. the filtered cells was uploaded (https://kleintools.hms.harvard.edu/tools/spring.html) per the guidence $^{14}$. Cell cluster information from Seurat analysis was also loaded for viewing.

For RNA velocity estimation, the bam file in 10x genomics output data was first re-counted with the Velocyto counting pipeline in python according to the manual ${ }^{16}$. The generated loom file was loaded to velocyto.R. Only counts of the 427 most variable genes were used for RNA velocity estimation. Finally, coordinates from Seurat's t-SNE analysis were used to embed the velocity results.

For gene regulatory network analysis, the $\mathrm{R}$ package GENIE3 ${ }^{29}$ was used. To simplify the results, only transcription-related genes in the 427 most variable genes were used to calculate gene-gene link matrix, and only the top 250 weighted link were adopted for visulation. To view the network, the $\mathrm{R}$ package igraph was used.

For Zipcode mapping, mean expression of all the genes in chosen cells was uploaded to www.itranscriptome.org/ according to the developer's guidence ${ }^{30}$.

\section{Identify genes required for ETV2-target gene activation}


In cell cluster 12, the cells were subset into two groups, one with Tal1 expression above the mean Tal1 level across the whole population, and the other with Tal1 below the mean level. In the first group, cells with gene $x$ expression above its mean level, while in the second group, cells with gene $x$ expression below its mean level, were counted seperately, and the sum of the two counts was divided by the total cell number of cluster 12 . This ratio was considered as the possibility of gene $x$ following Tal1. In fact, close gene-gene correlations can root from three possibilities, take Tal1 as an example: 1) gene $\mathrm{x}$ lies upstream of Tal1 and is required for its expression, 2) gene $\mathrm{x}$ is TAL1's target, 3) both gene $\mathrm{x}$ and Tal1 are ETV2's target, thus respond to ETV2 in a similar way. However, in the population where expression of Tal1 is just initiated, it is not likely that TAL1 activates its own target genes yet, therefore reduces possibility 2 . We can refer to a gene's expression pattern in the differentiation trajectory to test possibility 3. In fact, most of the genes tightly following Tal1 and Lmo2 were already extensively activated before ETV2 starts to function.

\section{Dye dilution assays}

Etv2-tdTomato ES cells ${ }^{18}$ is dissociated into single cell suspension and incubated with $1 \mu \mathrm{M}$ of VPD450 (BD Horizon ${ }^{\mathrm{TM}}$ ) in PBS for 10 minutes at room temperature. Then the cells were aggregated for normal differentiation.

\section{Microarray data analysis}

Wareing and colleagues' microarray data ${ }^{27}$ was re-analyzed using the Expression Console software (Affymetrix).

\section{Gene functional annotation}

Gene funcitonal annotation was performed using Metascape

(http://metascape.org/gp/index.html) ${ }^{31}$. 


\section{Flow cytometry}

Single cells were incubated with $\alpha$-mouse FLK1 (BioLegend) and $\alpha$-mouse PDGFR $\alpha$

(BioLegend). Data were acquired on LSR-Fortessa flow cytometer (BDbiosciences) and analyzed using the FlowJo (Treestar) software.

\section{Statistical analysis}

The results of flow cytometry were analyzed using Students' t test. $\mathrm{P}<0.05$ was considered significant.

\section{Data availability}

The data generated in this study can be downloaded from the NCBI Gene Expression Omnibus under accession number GSE130146. 
bioRxiv preprint doi: https://doi.org/10.1101/374629; this version posted June 3, 2019. The copyright holder for this preprint (which was not certified by peer review) is the author/funder. All rights reserved. No reuse allowed without permission.

\section{Acknowledgements}

We thank members of the Choi lab for helpful discussion and support. The work was supported

by NIH grant HL55337. 


\section{Figure legend}

Figure 1. scRNA-seq of EBs containing undifferentiated pluripotent cells and differentiated cells.

(A) Distribution of genes and transcripts detected in individual cells. (B) t-SNE projection of all cells. Cluster numbers is corresponding to those in supplemental Figure $1 \mathrm{~A}$. Potential lineage branches were annotated according to specific marker gene expression patterns. (CD) Expression of the indicated marker genes for specific cell states/lineage fates in the tSNE.

Figure 2. scRNA-seq captured continuous intermediates from naïve pluripotency to FLK1 derivatives.

(A) Re-clustering of populations in exit from naïve pluripotency and bifurcation of endoderm and mesoderm. Clusters 4, 5, 8, 9, 11 and 17 in Figure 1B were picked to re-cluster based on Principle Component Analysis. The first two components are shown. The clusters are in the same colors as they are in Figure 1B. "naïve", naïve pluripotency. (B) Cells in the endoderm differentiation route (red dash line-surrounded region) were ordered into pseudotime line. Arrows indicate the differentiation direction. Heatmap of naive pluripotent cellenriched genes are shown. Brown box encircles genes heterogeneously expressed in the naïve stage; blue box marks the constantly expressed naïve stage-specific genes. (C) Barplot of expression of selected marker genes along the endoderm pseudotime line. Shaded region covers cells maintaining high Dppa3 expression in the naïve population. Blue dash line marks the exit point from naïve pluripotency. (D) Barplot of expression of marker genes along the pseudotime line of endoderm differentiation. Red dash line marks the point, where endoderm fate is committed. (E) Expression of indicated genes in early mesoderm cells. (F) Zipcode mapping of early mesoderm cells (cluster 4 in Figure 1B. Mean values of each genes in the cluster were used for the mapping) to E7.0 mouse embryo. (G-H) 
Expression of indicated genes. (I) scheme of development routes of indicated mesoderm lineages.

Figure 3. Cells corresponding to different development stages proliferate equally.

(A) Expression of indicated cell cycle genes. (B) Assigned cell cycle phases of each cells. (C) Proliferation of cells in EBs treated with or without $2 \mu \mathrm{M}$ of ERK inhibitor PD0325901 from day 3 was compared based on dilution of the Dye VPD450. Dye dilution and the Etv2-tdTomato reporter gene expression were examined 24 hours later using flow cytometry.

\section{Figure 4. Underlying transcription factor modules in hemangiogenic lineage development.}

(A) Re-clustering of populations in the route of hemangiogenic lineage development. Clusters 3, 4, 8, 9, 10, 12 and 17 in Figure 1B were picked to re-cluster. The first two components of PCA are shown for re-clustered cells. The clusters are in the same colors as they are in Figure 1B. Arrows indicate the differentiation direction. Expression of the representative marker genes in the re-clustered populations is shown. (B) The re-clustered cells are ordered into a pseudo-time line of differentiation. Expression dynamics of representative marker genes along the pseudo-time line is shown. Gene expression data was smoothened. The expression curves and the gene names are in same colors. At the bottom, color bars indicate the cells' original identities as in Figure 1B. Cell states were annotated: "naïve, naïve pluripotent state; "mesendo", mesendoderm; "early meso", early mesoderm; "FLK1 meso", FLK1 mesoderm; "hemangio", hemangiogenic lineage. (C) Dynamics of transcription-related factors from the 427 most variable genes is shown along the pseudo-time line of hemangiogenic lineage development. The genes are hierarchically clustered based on Pearson's correlation. Boxes in a yellow dash-line indicate the obvious stage-specific gene modules. (D) Gene regulatory network of the trancription-related factors shown in Figure 4C. 
Only the top250 gene-gene links were shown. The width of the edges indicated the weight of the links. (E) Gene expression similarities among individual clusters along the hemangiogenic development route. The mean values of gene expression of cells in individual clusters were used to calculate the Pearson's correlation scores. (F) Scheme of the 'relay'like transcription factor modules underlying FLK1 development.

\section{Figure 5. ETV2 triggers a sensitive switch initiating hemangiogenic program,}

\section{depending on cell adhesion signaling}

(A) ETV2 target genes sensitively respond to Etv2 dosage above the threshold. The input data was from smoothed and re-scaled expression of related genes along the pseudo-time line of hemangiogenic lineage development. Datasets only before Etv2 achieves its highest level were displayed, because after that Etv2 starts to be down regulated. (B) Scheme for identification of potential regulators of ETV2-mediated hemangiogenic fate specification. If gene $B$ is required for gene $A$ expression, then in a cell where gene $A$ is expressed gene $B$ should also be expressed. In a population, more gene A expressing-cells have gene B expression, it is more likely that gene B is required for gene A expression. (C) All detected genes' relationship with Tal1. $\mathrm{x}$-axis is the possibility of Tal1 expression requires a given gene $x$ in group 12 in Figure 1C, where Etv2 achieves threshold expression and starts to activate hemangiongenic genes. $y$-axis is the possibility of Tal1 in all cells in return is required for a specific gene's expression. Etv2 and Kdr/Flk1, two known upstream regulators of Tal1, are labeled in the plot. (D) Comparison of genes required for Tal1 expression to that required for Lmo2 or Eomes. Eomes is a FLK1 mesoderm-expressing transcription factor but not reported regulating hemangiogenic genes. For the top 100 genes that most tightly required for Tal1 expression in the cluster 12 (dots in shadow regions), the Pearson's correlation coefficient $(r)$ between x-axis and y-axis are shown respectively. Genes of interest are shown in color. House keeping genes Actb (beta-actin) and Gapdh are shown as control. 
(E) A2lox ES cells with DOX-inducible ETV2-mCherry were transferred to feeder-free condition and cultured in the presence of $0.5 \mu \mathrm{g} / \mathrm{mL}$ of DOX, simultaneously treated with or without PP2 $(5 \mu \mathrm{M})$ for 24 hours. Cells were then analyzed for FLK1 and mCherry levels using flow cytometry. The horizontal red lines mark ETV2 threshold. The statistics summary is shown on the right. ${ }^{* *}, \mathrm{P}<0.001$.

Figure 6. 'Default' vascular smooth muscle fate of FLK1 mesoderm.

(A) Left, reclustering of populations in the routes of VSM and hemangiogenic lineage development. Clusters 0, 3, 4, 8, 9, 10, 12, 13 and 17 in Figure 1B were picked, and the first two components of PCA are shown. Right, pseudotime analysis of the reclustered cells. (B) The comparison of the most variable 427 genes' dynamics along the VSM or hemangiogenic routes is shown. The genes were clustered based on Pearson's correlation. Color bars indicate cells' original identities. "a", "b", and "c", shown in a yellow dash-box, mark three groups of genes that are mostly enriched in the VSM branch. (C) Expression dynamics of indicated genes along the pseudo-time lines of hemangiogenic and VSM lineage development is shown. The expression values were smoothened. The colors of curves and gene names are consistent. (D) Clusters 0, 3, 10, 12 and 13, corresponding to the VSM/hemangiogenic lineage bifurcation fork in Figure 1B were picked and reclustered. Cells are colored according to the expression levels of indicated genes. (E) Violin plots comparing indicated genes' expression in different populations. ${ }^{* *}, \mathrm{P}<0.01$; ${ }^{* * *}, \mathrm{P}<0.001$. (F) Important VSM transcription factors respond to Etv2 overexpression. Day 2.5 Etv2-knockout EBs were induced for exogenous Etv2 expression using DOX. Two replicates of samples corresponding to DOX induction for 0 hour, 12 hours or 24 hours were collected for microarray analysis. 
Figure 7. Characteristics of FLK1 mesoderm are enriched in vascular smooth muscle tissue in vivo.

(A) t-SNE plot of scRNA-seq analysis of yolk sac. (B) LPM-score gene expression in E8.5 yolk sac cells. (C) Expression of indicated genes in E8.5 yolk sac. (D) Re-analysis of lbarraSoria et al.'s ${ }^{5}$ scRNA-seq of E8.25 embryo. Only mesoderm tissues were shown. (E-F) Expression of indicated genes in Ibarra-Soria et al.'s mesoderm population. (G) Comparison of VSM marker genes from indicated datasets. (H) Annotation of the shared 72 VSM genes by the three different datasets. (I) Model of gene module dynamics along VSM and hemangiogenic lineage development. 
A

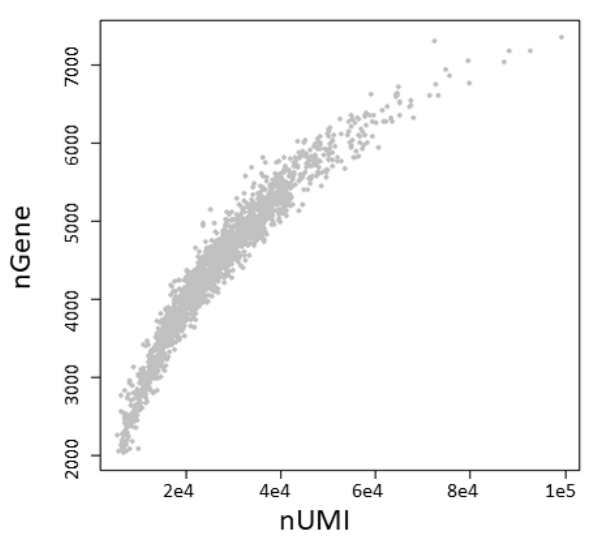

C

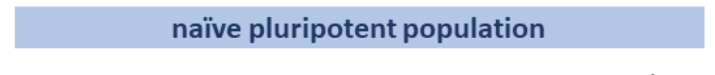

B

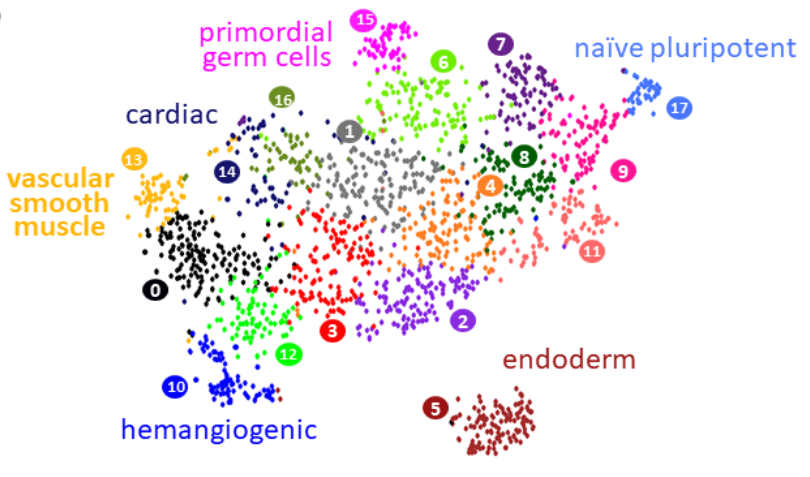

FLK1 mesoderm and hemangiogenic lineage

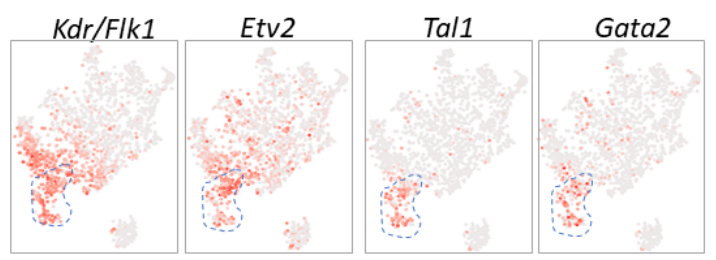

low high
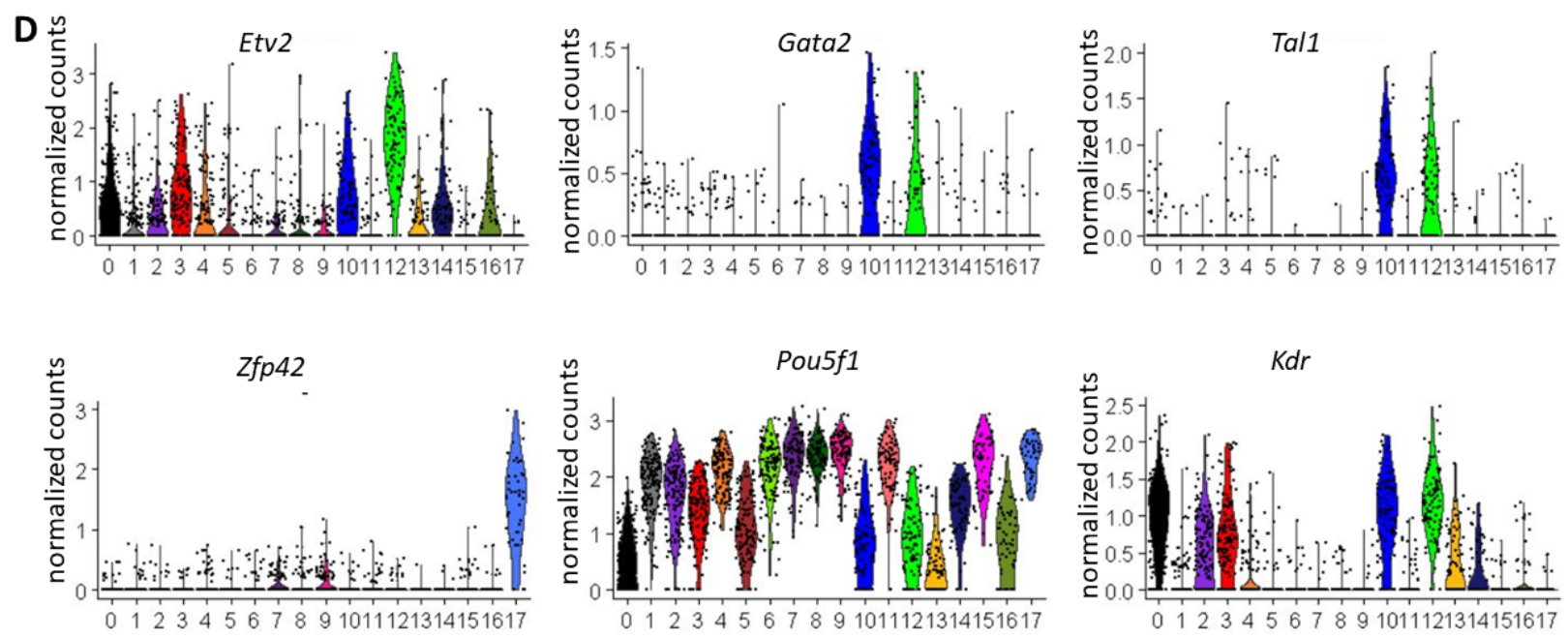

\section{Figure 1. EBs contain both undifferentiated pluripotent cells and FLK1 mesoderm tissues.}


bioRxiv preprint doi: https://doi.org/10.1101/374629; this version posted June 3, 2019. The copyright holder for this preprint (which was not certified by peer review) is the author/funder. All rights reserved. No reuse allowed without permission.

A

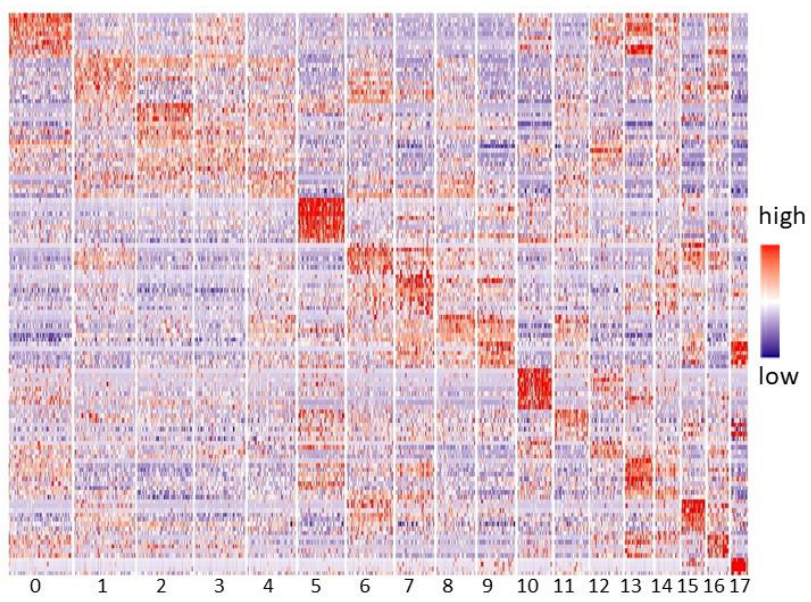

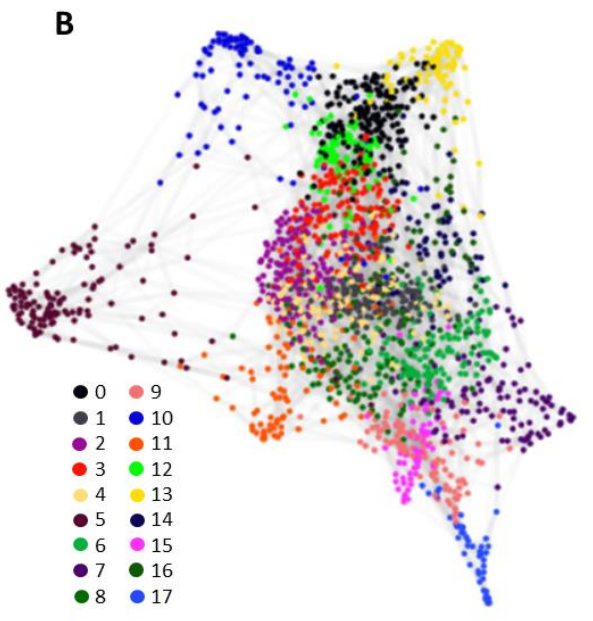

Figure 1S 
A

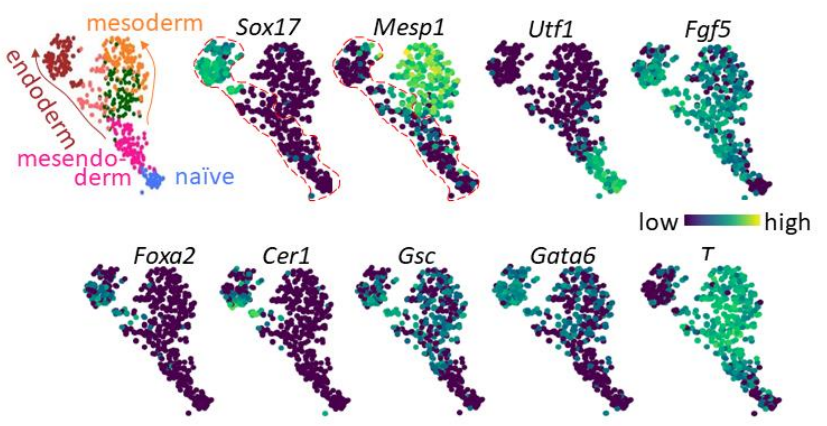

B Loss of pluripotency in endoderm development route

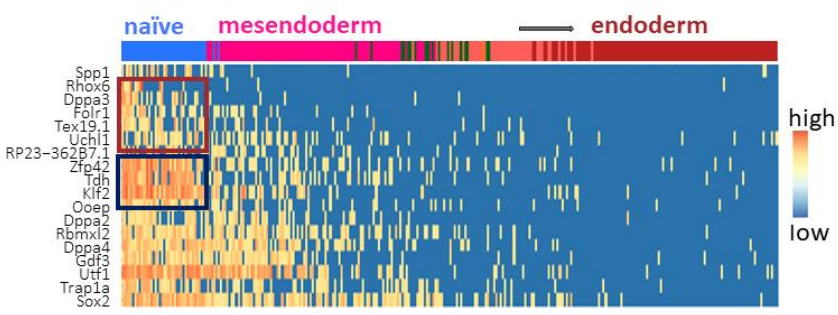

E

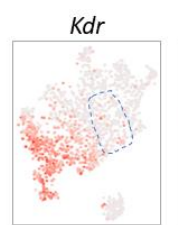

$\mathbf{F}$

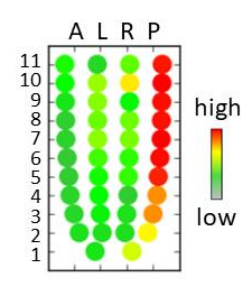

Pdgfra

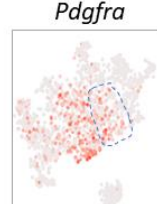

G

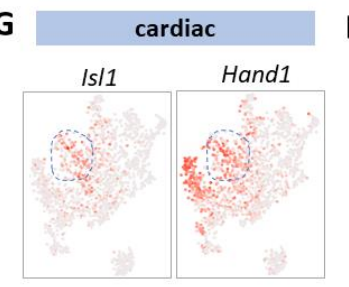

C

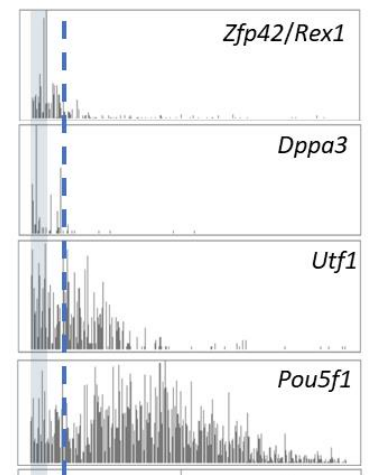

iv

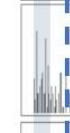

I

I
Nanog

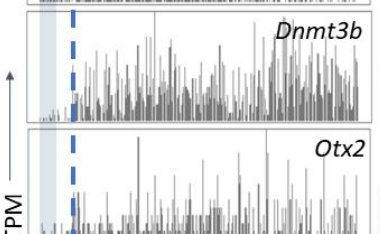

D
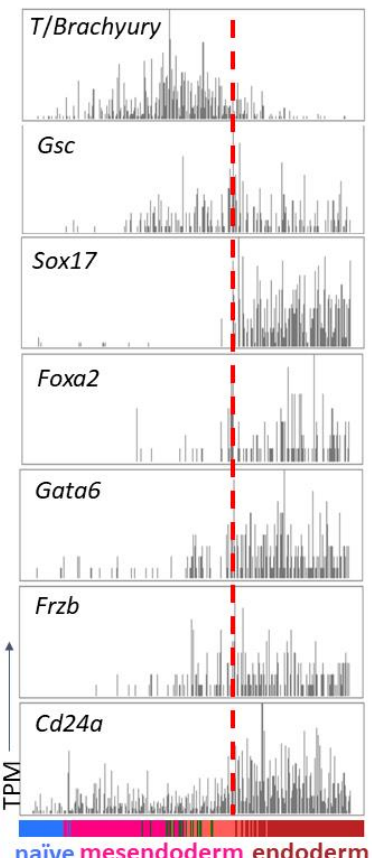

naïve mesendoderm endoderm naïve mesendoderm endoderm

\begin{abstract}
\begin{tabular}{c|c} 
Tbx6 Mesp1 & $T$
\end{tabular}
\end{abstract}

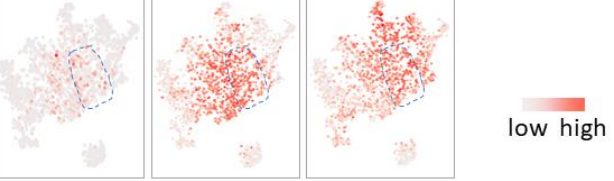

H vascular smooth muscle

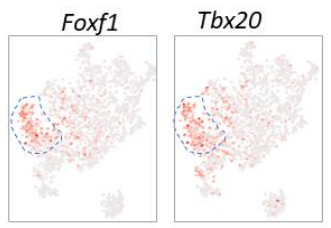

I

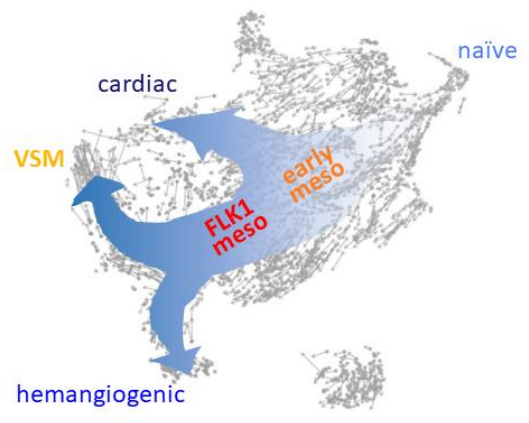

Figure 2. scRNA-seq captured continuous intermediates from naïve pluripotency to FLK1 derivatives. 
bioRxiv preprint doi: https://doi.org/10.1101/374629; this version posted June 3, 2019. The copyright holder for this preprint (which was not certified by peer review) is the author/funder. All rights reserved. No reuse allowed without permission.

A

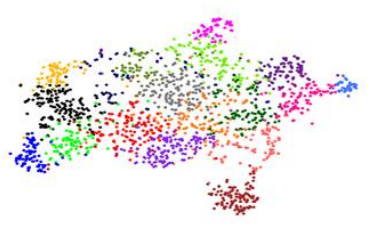

C endoderm development -

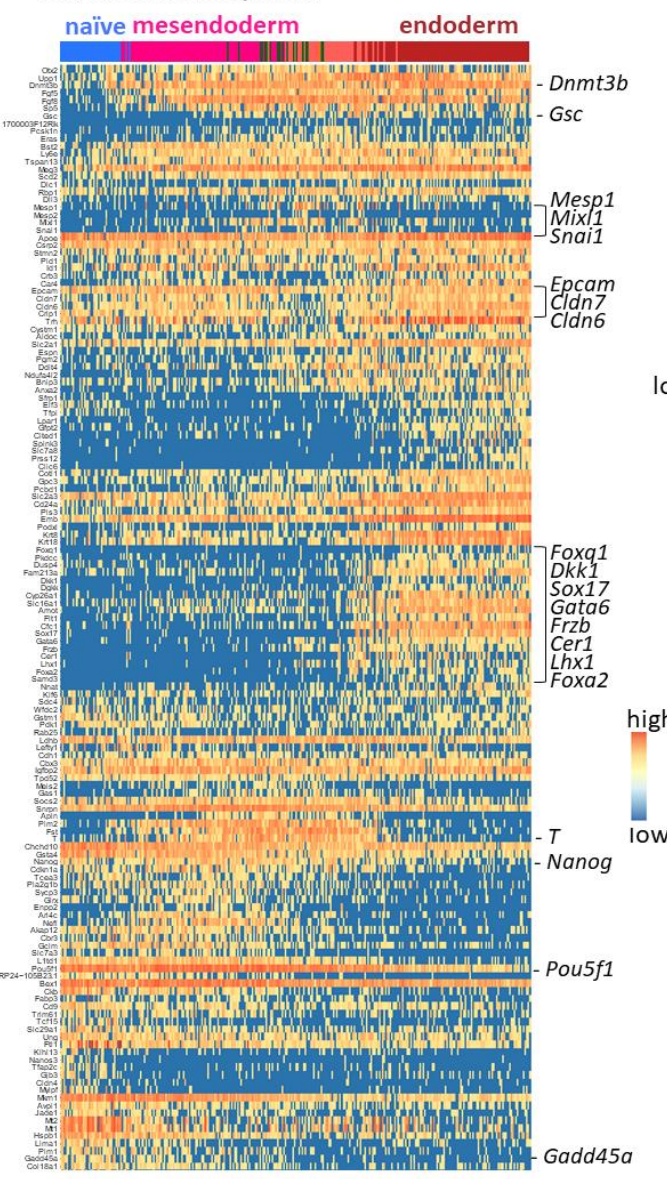

B

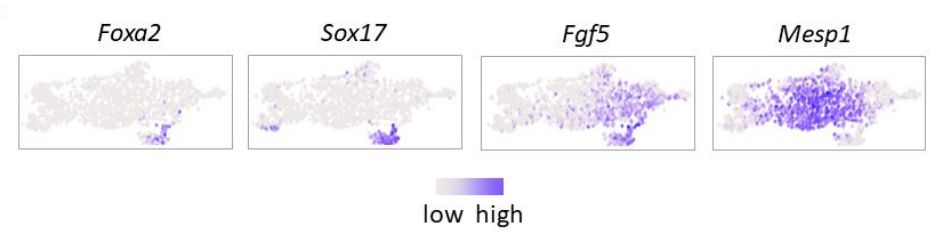

D

E6.75 Mesp1-labeled cells (Lescroart et al.)
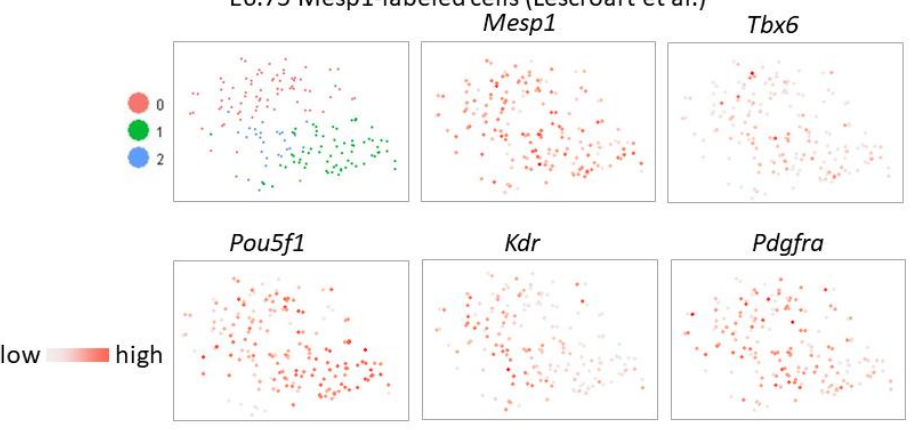

E

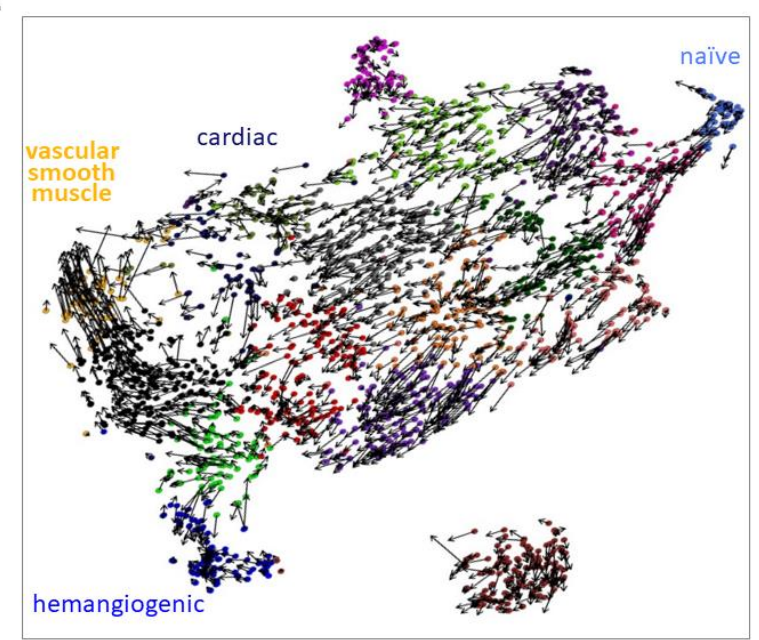

Figure 2S 
A
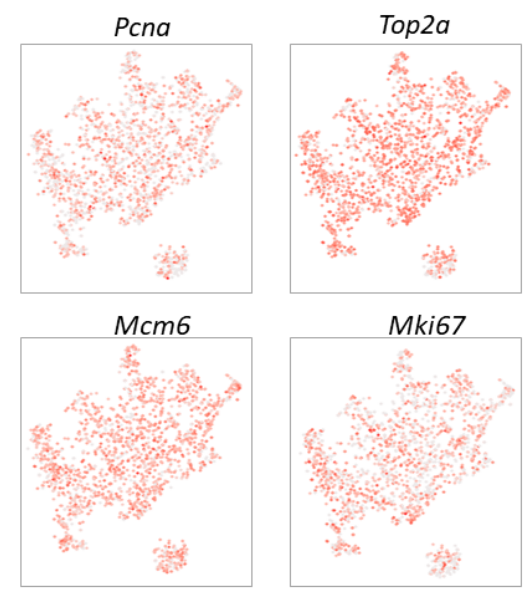

Mki67

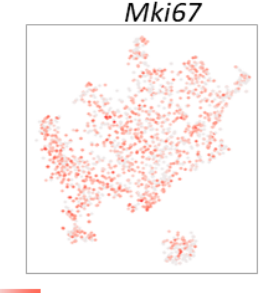

low high
B

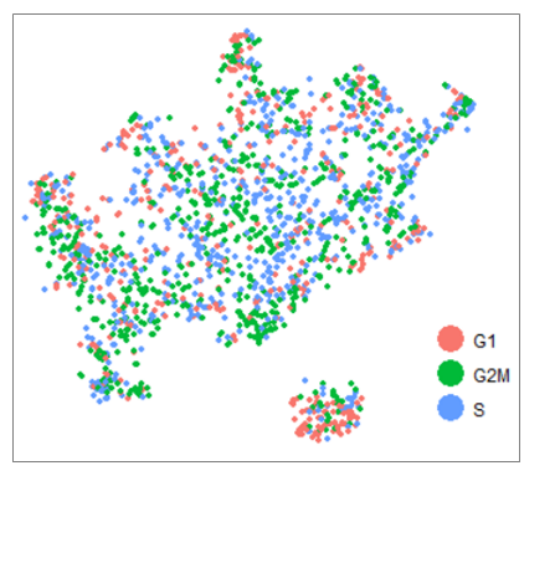

C

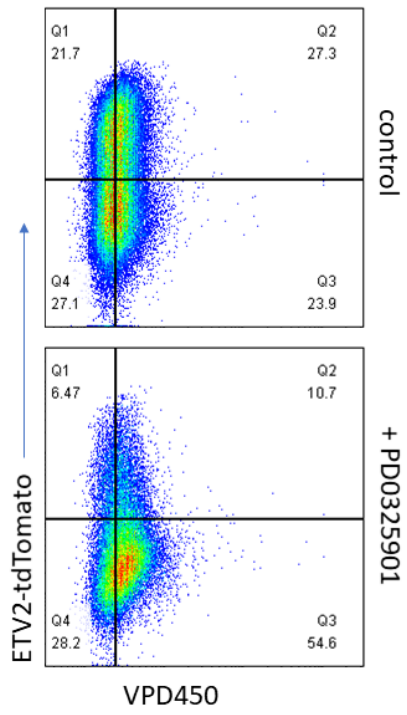

Figure 3. Cells corresponding to different development stages proliferate equally. 
A
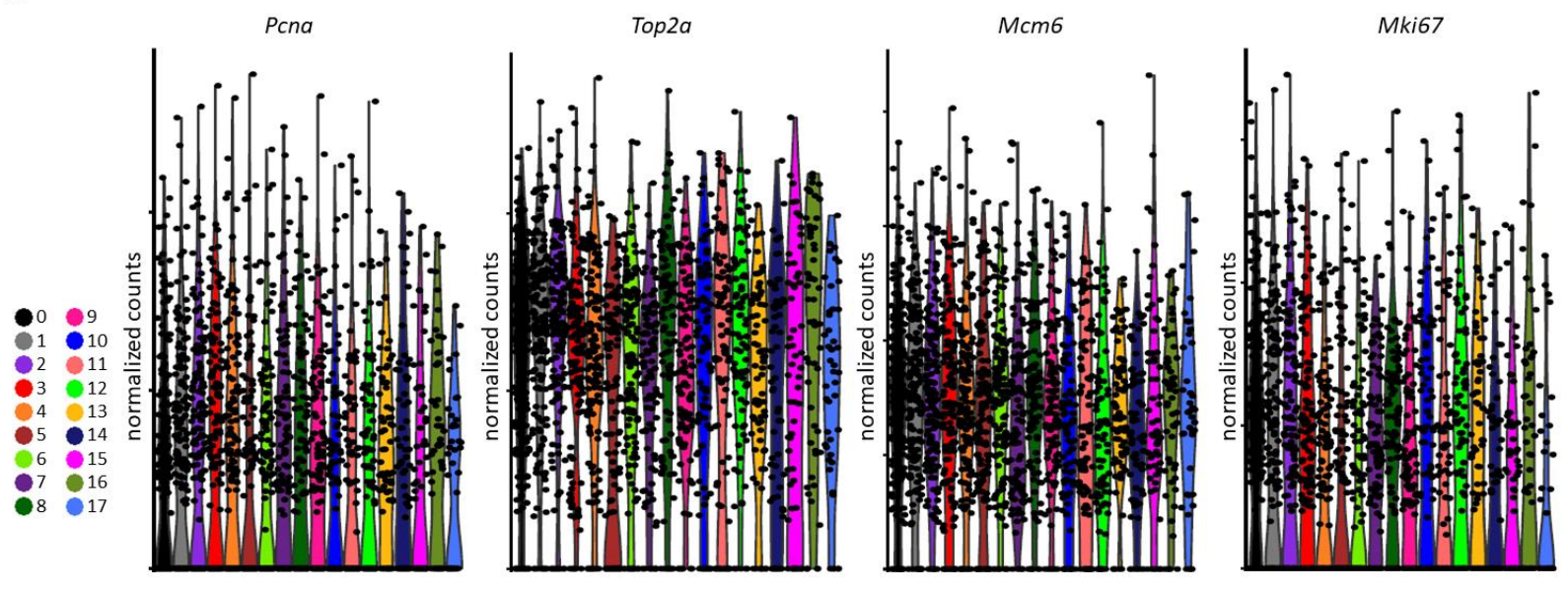

B

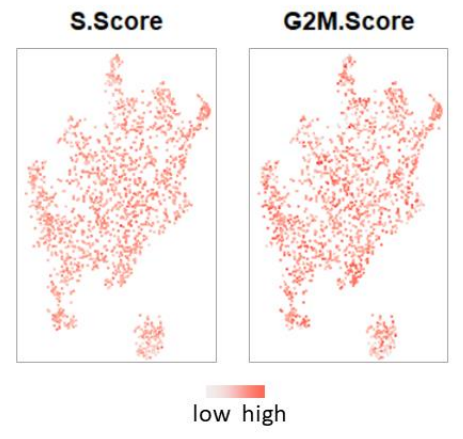

Figure 35 
A hemangiogenic lineage development route

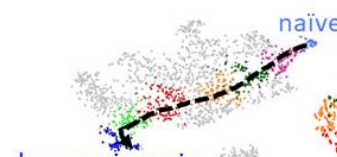

hemangiogenic
Pou5f1
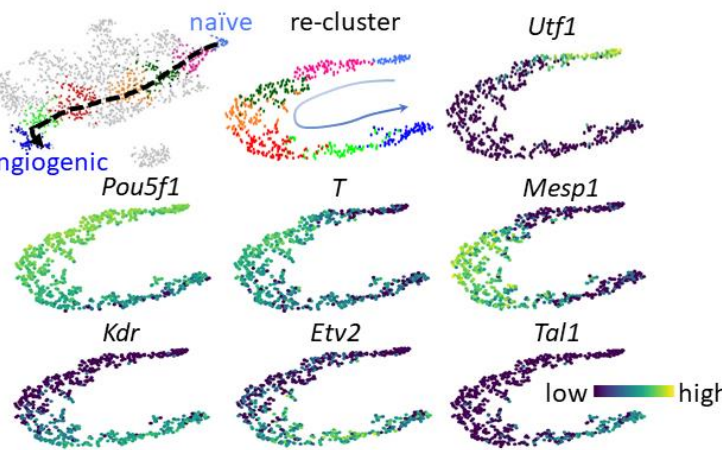

C

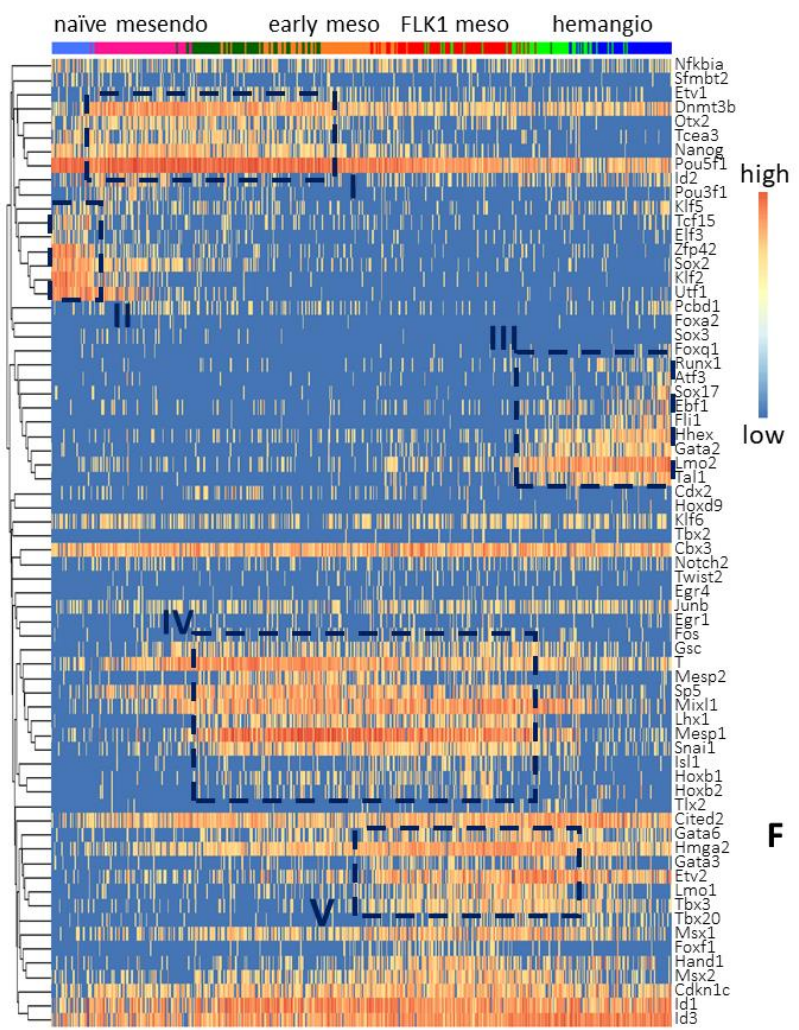

B

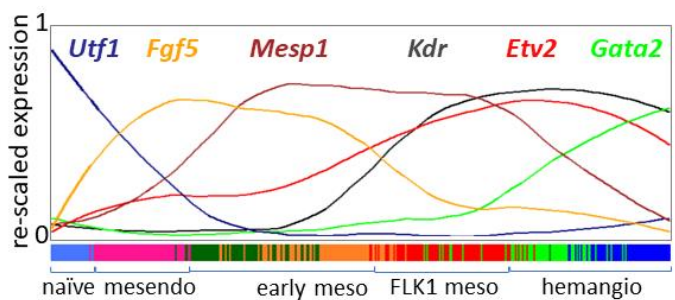

D

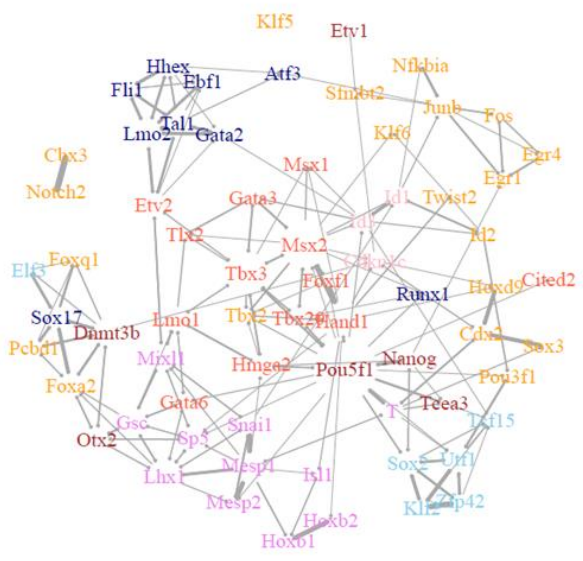

E

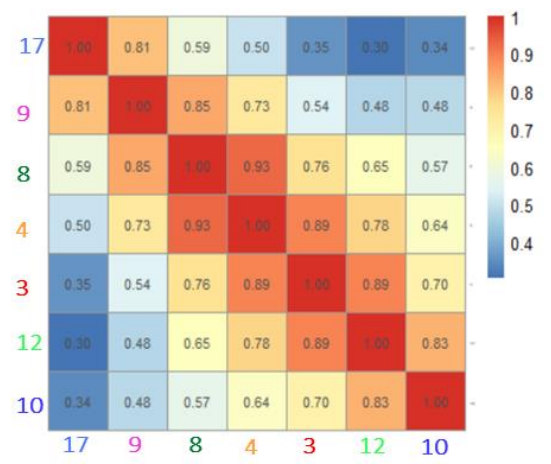

Relay of transcription facotor modules:

mesendoderm $\rightleftarrows$ early mesoderm $\rightleftarrows$ FLK1 mesoderm

Figure 4. Transcription factor modules underlying hemangiogenic lineage development. 
bioRxiv preprint doi: https://doi.org/10.1101/374629; this version posted June 3, 2019. The copyright holder for this preprint (which was not certified by peer review) is the author/funder. All rights reserved. No reuse allowed without permission.

A

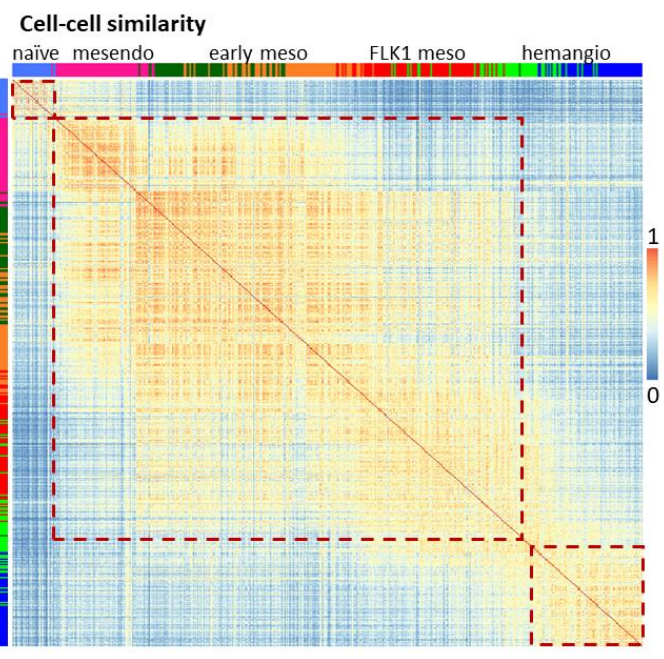

B

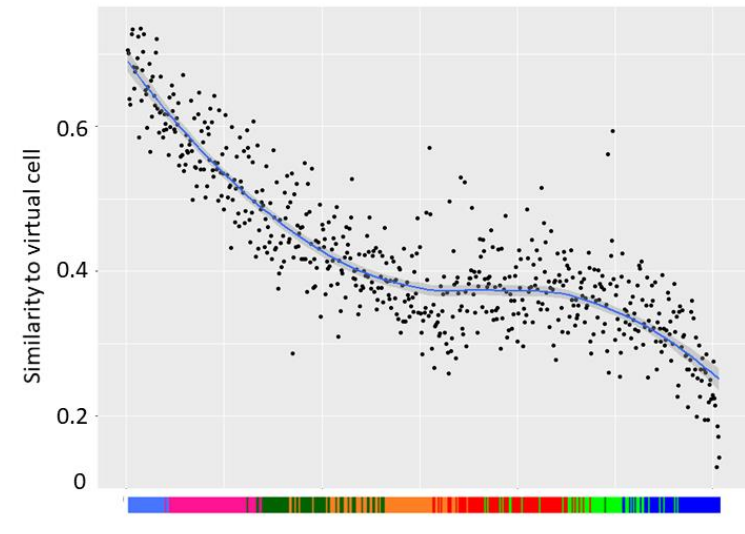

Figure 4S 
A

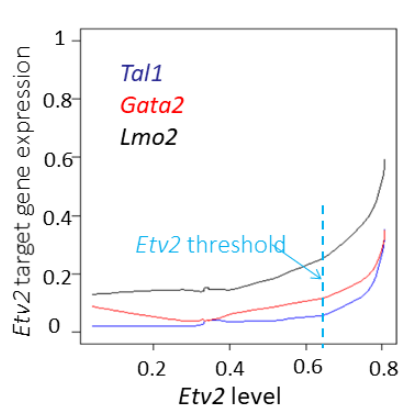

B

gene: $\nabla$ OFF $\nabla$ ON

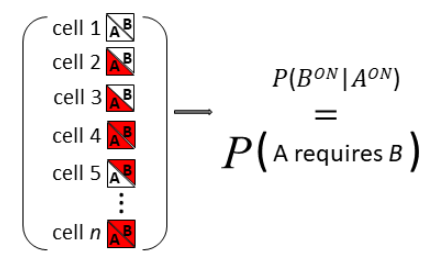

D

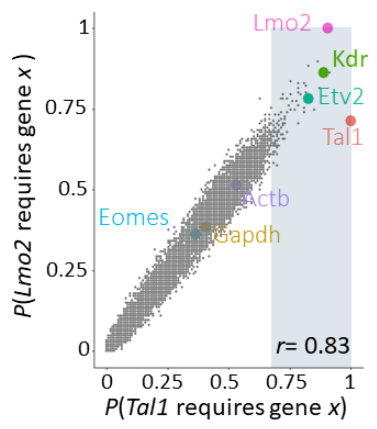

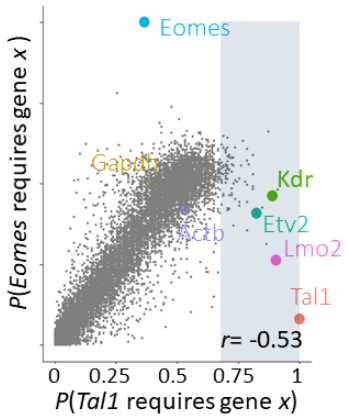

$\mathbf{E}$
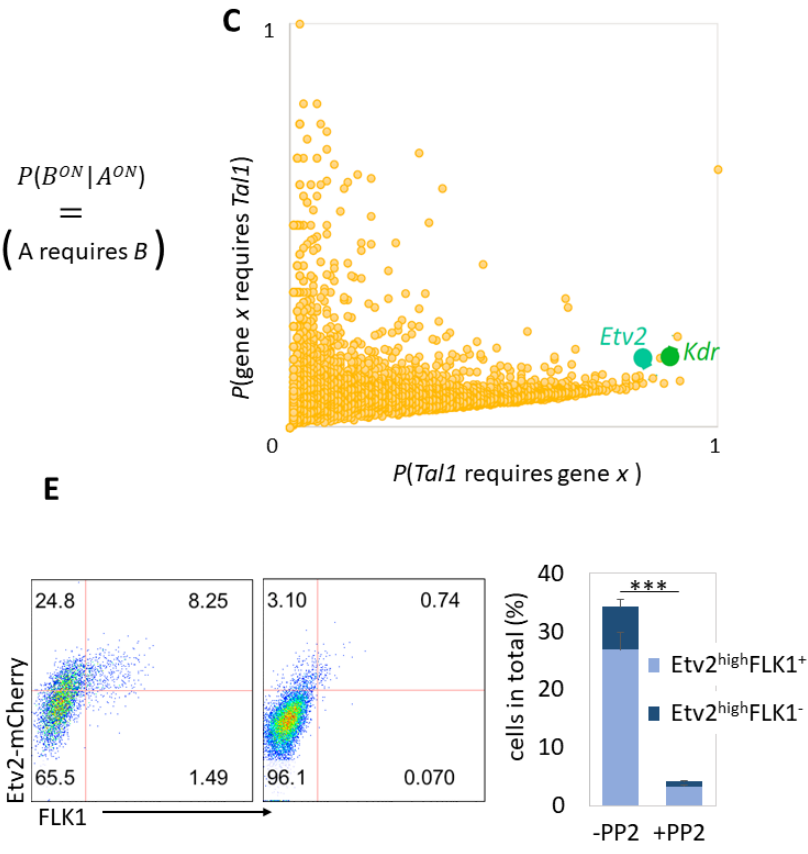

Figure 5. Etv2 triggers a sensitive switch initiating hemangiogenic program, depending on cell adhesion signaling 
bioRxiv preprint doi: https://doi.org/10.1101/374629; this version posted June 3, 2019. The copyright holder for this preprint (which was not certified by peer review) is the author/funder. All rights reserved. No reuse allowed without permission.

A

Rap1 signaling pathway

Platelet activation, signaling and aggregation cAMP signaling pathway cell junction organization endothelial cell differentiation GRB2:SOS to MAPK signaling for Integrins p130Cas to MAPK signaling for integrins cell junction assembly endothelium development Leukocyte transendothelial migration Platelet activation RAF/MAP kinase cascade MAPK1/MAPK3 signaling Integrin signaling MAPK family signaling cascades Platelet Aggregation (Plug Formation) establishment of endothelial barrier MAP2K and MAPK activation MET promotes cell motility Focal adhesion

B

$-\log 10(P) \quad 0$ 5 10

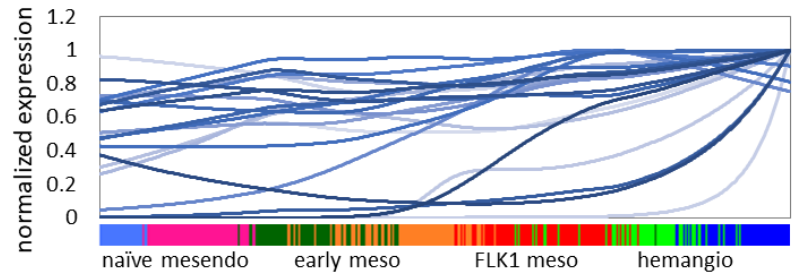

C

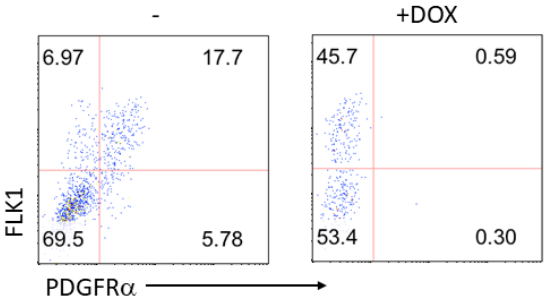

D

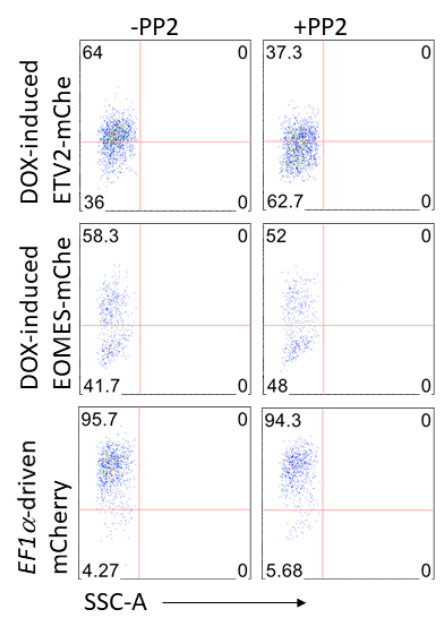

$+\mathrm{DOX}+\mathrm{PP} 2$

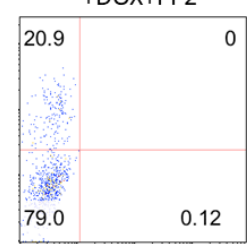

100

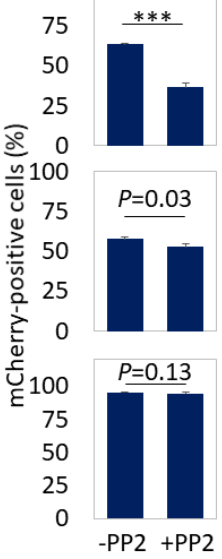

Figure 5S 
A

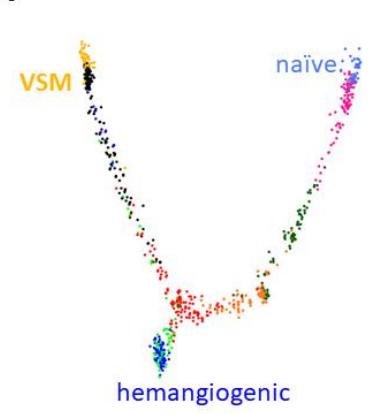

C

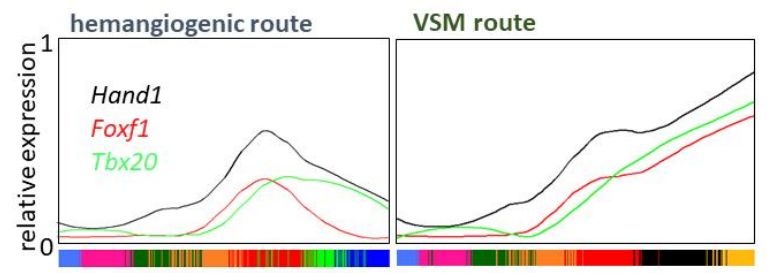

D

VSM vs. hemangiogenic fates bifurcation

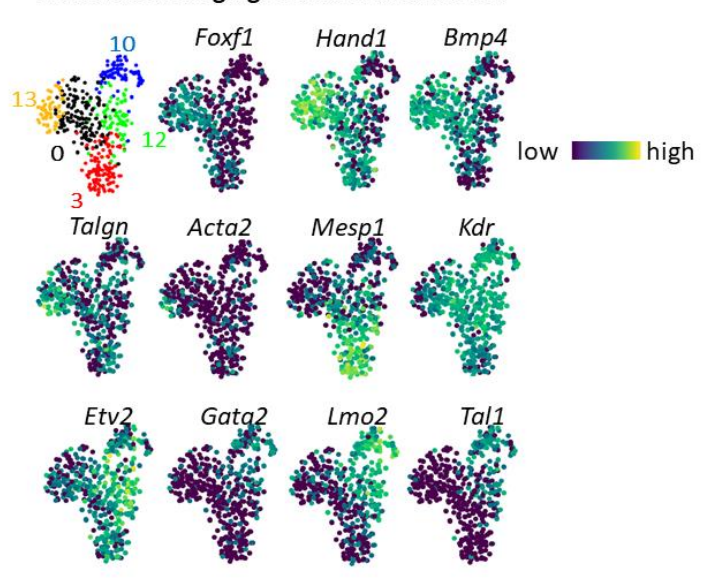

B

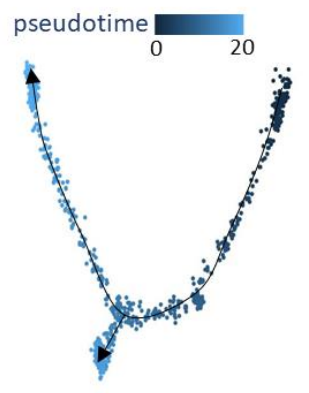

E

$\mathbf{F}$
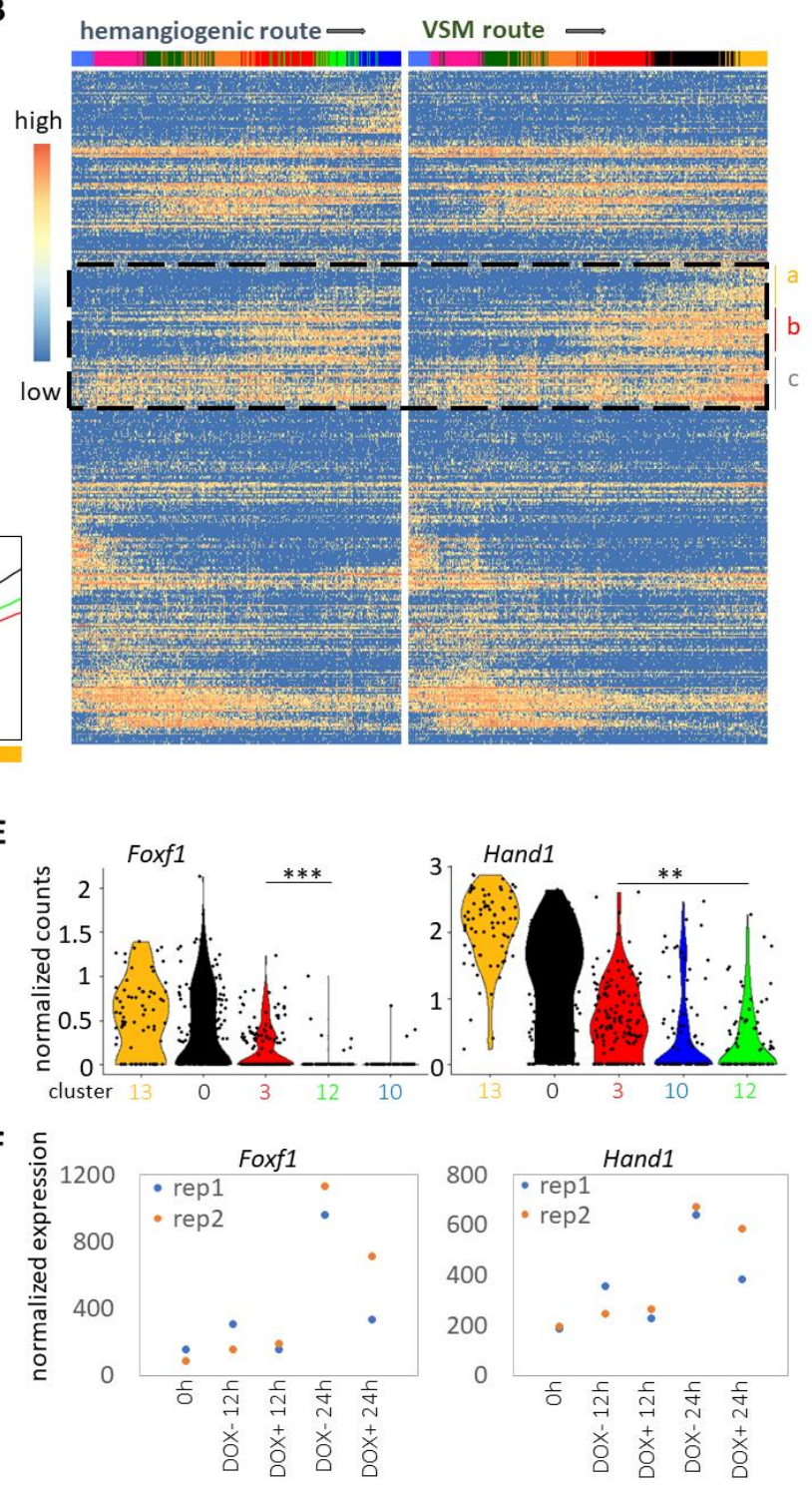

Wareing et al.

Figure 6. FLK1 mesoderm is destinied to vascular smooth muscle lineage, while ETV2 skews it into hemangiogenic fate. 
bioRxiv preprint doi: https://doi.org/10.1101/374629; this version posted June 3, 2019. The copyright holder for this preprint (which was not certified by peer review) is the author/funder. All rights reserved. No reuse allowed without permission.

A

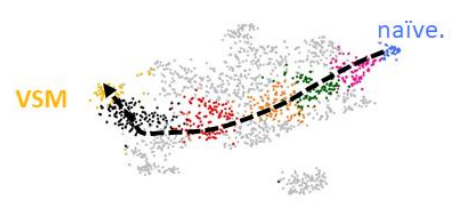

B

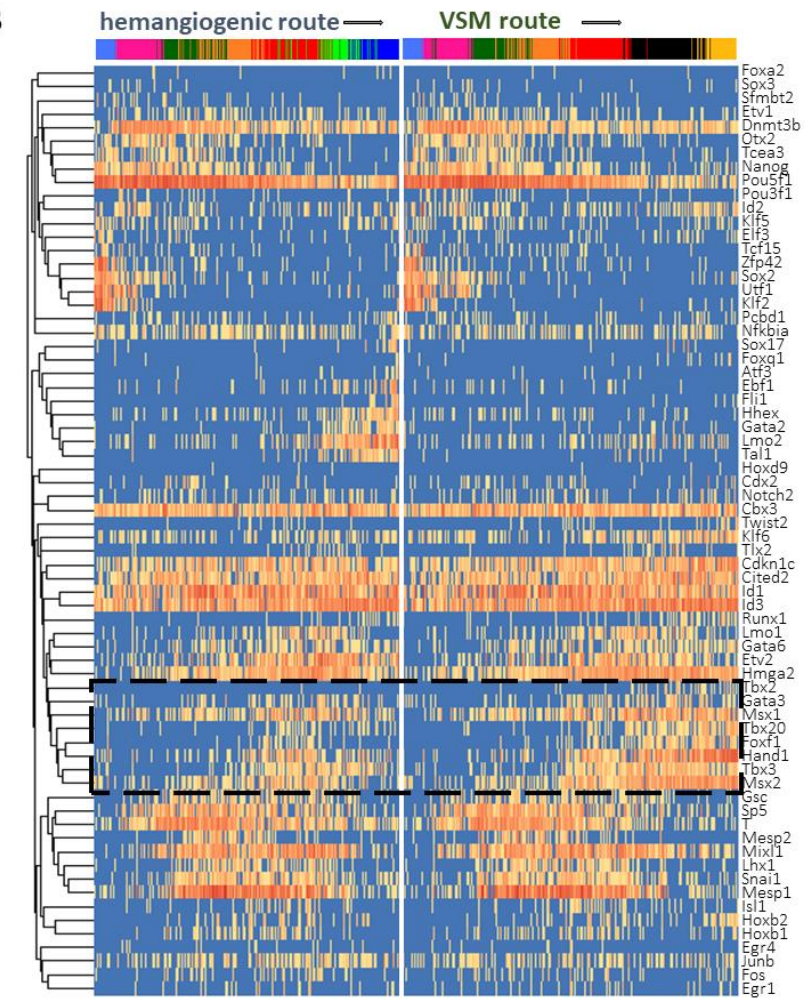

Figure 6S 
A

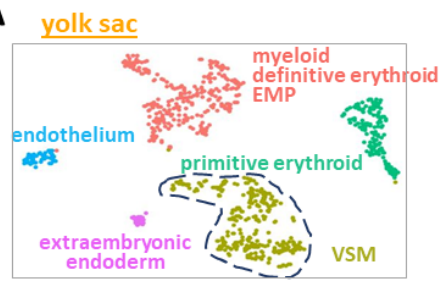

D embryonic mesoderm tissues (Ibarra-Soria et al.)

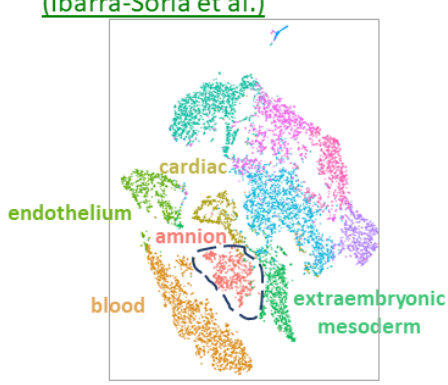

E

B
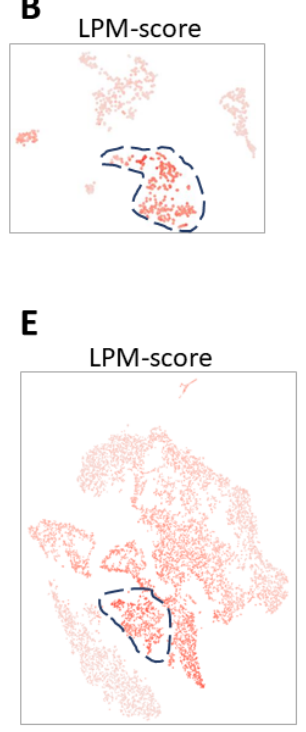

H

actin filament-based process
Smooth Muscle Contraction Smooth Muscle Contraction
muscle structure development muscle structure development
positive regulation of EMT positive regulation of EMT
cardiac chamber development positive regulation of cell migration collagen biosynthetic process RHO GTPases activate PAKs Focal adhesion
RHO GTPases activate IQGAPs negative regulation of neurogenesis
Hemostasis Striated Muscle Contraction
integrin-mostasis integrin-mediated signaling pathway maintenance of protein location branching morphogenesis of an epithelial tube tumor necrosis factor-mediated signaling pathway
Regulation of insulin secretion

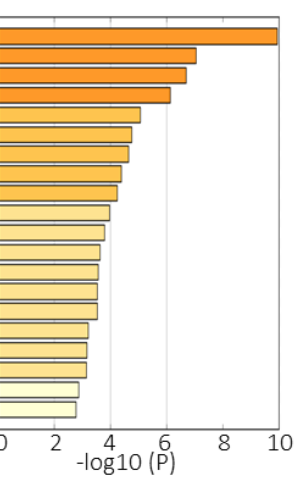

C

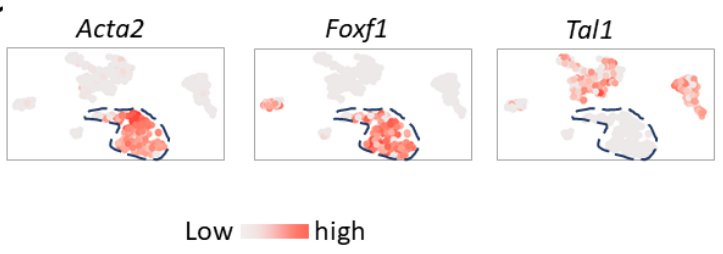

F
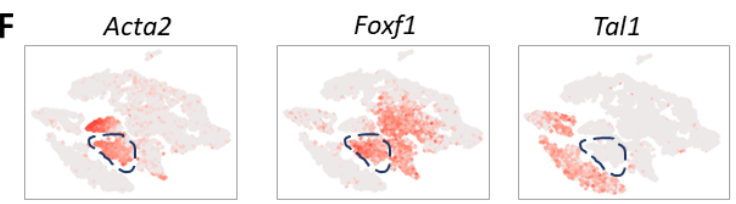

hemangiogenic route

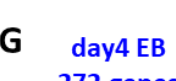

272 genes

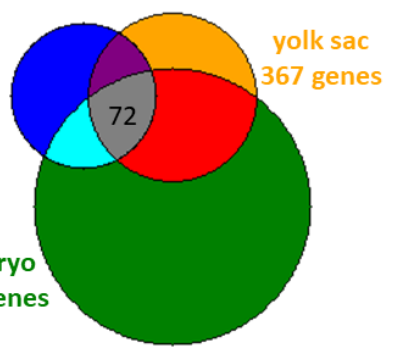

VSM route

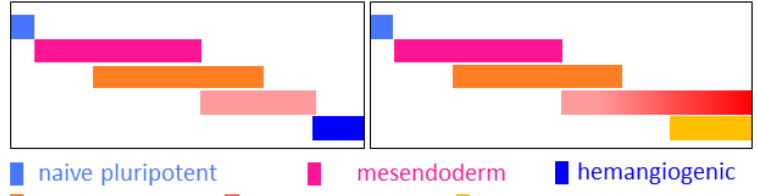

_ early mesoderm FLK1 mesoderm

r smooth muscle

embryo 966 genes

Figure 7. FLK1 mesoderm characteristics are enriched in vascular smooth muscle tissue in vivo. 
A

day4 EB

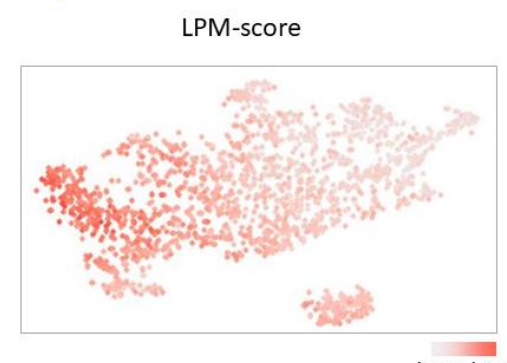

low high
C

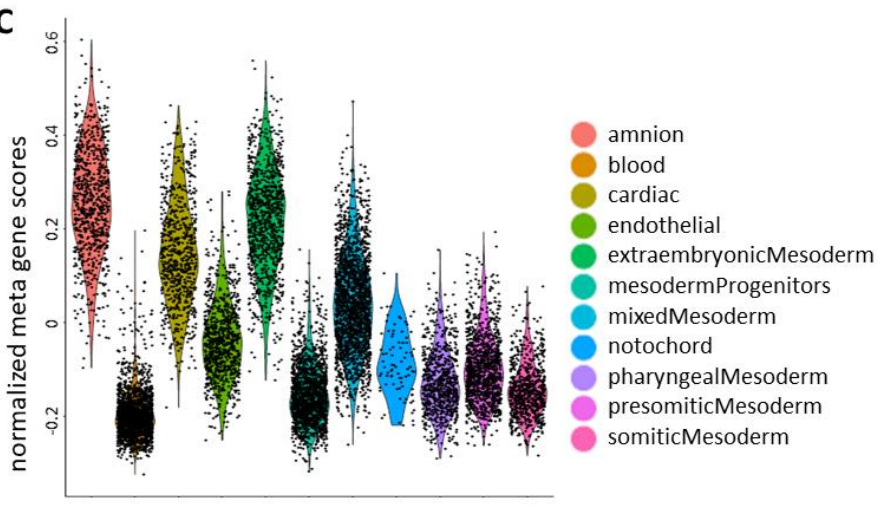

B

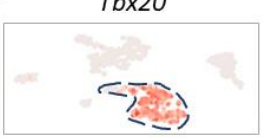

Msx1

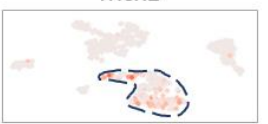

Hand1

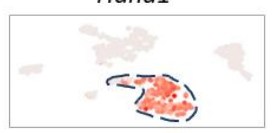

Msx2

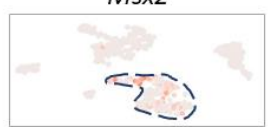

D

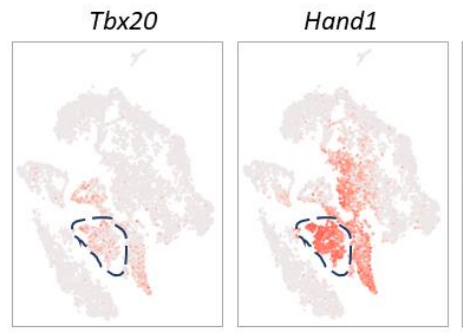

Msx1

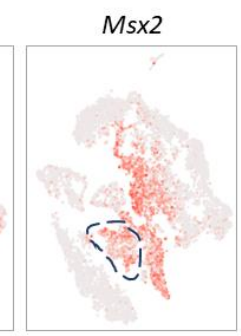

Figure 7S 


\section{Supplemental Figure legends}

\section{Figure 1S.}

(A) Representative marker gene expression in each cluster. Only at most 10 representative marker genes are displayed for each cluster. (B) SPRING plot of the single cell transcriptome. Each cell is in the same color as they are in Figure 1B.

\section{Figure 2S.}

(A) Dynamics of the 163 most variable genes along the endoderm development route. The genes were clustered based on Pearson's correlations. (B) t-SNE plot of the cells embedded with marker genes selected based on less strengency. The cells' colors show their original identities in Figure 1B. (C) Gene expression in the new t-SNE plot. (D) Expression of indicated genes in E6.75 Mesp1-labeled cells (re-analysis of Lescroart et al.'s ${ }^{8}$ data). (E) RNA velocity estimation of the cells. The arrows indicate estimated differentiation trends of given cells. The colors of the cells is same to that in Figure 1B.

\section{Figure 3S.}

(A) VIn plot of cell cycle genes in different clusters. (B) Cell cycle scores of each cells.

\section{Figure 4S.}

(A) Cell-cell Pearson's correlations along the pseudo-time line based on expression of 427 most variable genes. The brown dash boxes mark the three relatively isolated naïve pluripotent population, 'gastrulation' stage, and hemangiogenic lineage. (B) The first 20 cells in the hemangiogenic lineage route were picked and their mean expression was treated as a virtual cell. Then each cell's similarity in the development route to this virtual cell was indicated in the dot plot. Bars at the bottom show each cell's original identity. 


\section{Figure 5S.}

(A) Top 100 genes that are most required for Tal1 expression and the top 100 most required for $L$ mo2 expression have 66 overlap. These 66 genes were analyzed for functional enrichment. (B) Expression of 22 cell adhesion-related genes along the pseudo-time line of hemangiogenic lineage development. The expression values were smoothened for visualization. (C) A2lox ES cells with DOX-inducible exogenous Etv2 expression were differentiated in serum medium for 3 days, EBs were then treated with or without the SRC kinase inhibitor PP2 $(5 \mu \mathrm{M})$ for another 24 hours in the presence of $2 \mu \mathrm{g} / \mathrm{mL}$ of DOX. EBs without PP2 or DOX treatment were used as control. EBs were collected and analyzed using flow cytometry for surface marker examination. (D) A2lox ES cells with DOX-inducible ETV2mCherry or EOMES-mCherry expression, or EF1 $\alpha$-driven constitutive mCherry expression were cultured in ES cell medium, treated with or without the SRC kinase inhibitor PP2 $(5 \mu \mathrm{M})$ in the presence of $0.5 \mu \mathrm{g} / \mathrm{mL}$ of DOX for 1 days. Cells were collected and analyzed using flow cytometry for mCherry and FLK1 expression. The statistics summary is shown on the right. ***, $\mathrm{P}<0.001$.

\section{Figure 6S.}

(A) Populations selected for VSM development route reconstitution. (B) Comparison of expression dynamics of transcription-related factors along VSM vs. hemangiogenic lineage development. The genes were clustered based on the Pearson's correlations.

\section{Figure 7S.}

(A) LPM-score gene expression in day 4 EB cells. (B) Expression of indicated genes in E8.5

yolk sac cells. (C) VIn plot of FLK1-meata gene expression in different mesoderm tissues from E8.25 embryo. (D) Expression of indicated genes in E8.25 embryonic mesoderm cells. 


\section{References}

1. Lugus JJ, Park C, Ma YD, Choi K. Both primitive and definitive blood cells are derived from Flk-1+ mesoderm. Blood. 2009;113(3):563-566.

2. Yamashita J, Itoh $\mathrm{H}$, Hirashima $\mathrm{M}$, et al. Flk1-positive cells derived from embryonic stem cells serve as vascular progenitors. Nature. 2000;408(6808):92-96.

3. Motoike T, Markham DW, Rossant J, Sato TN. Evidence for novel fate of Flk1+ progenitor: contribution to muscle lineage. Genesis. 2003;35(3):153-159.

4. Pijuan-Sala B, Griffiths JA, Guibentif C, et al. A single-cell molecular map of mouse gastrulation and early organogenesis. Nature. 2019;566(7745):490-495.

5. Ibarra-Soria X, Jawaid W, Pijuan-Sala B, et al. Defining murine organogenesis at single-cell resolution reveals a role for the leukotriene pathway in regulating blood progenitor formation. Nat Cell Biol. 2018;20(2):127-134.

6. Farrell JA, Wang Y, Riesenfeld SJ, Shekhar K, Regev A, Schier AF. Single-cell reconstruction of developmental trajectories during zebrafish embryogenesis. Science. 2018.

7. Mohammed H, Hernando-Herraez I, Savino A, et al. Single-Cell Landscape of Transcriptional Heterogeneity and Cell Fate Decisions during Mouse Early Gastrulation. Cell Rep. 2017;20(5):1215-1228. 8. Lescroart F, Wang X, Lin X, et al. Defining the earliest step of cardiovascular lineage segregation by single-cell RNA-seq. Science. 2018;359(6380):1177-1181.

9. Scialdone A, Tanaka Y, Jawaid W, et al. Resolving early mesoderm diversification through single-cell expression profiling. Nature. 2016;535(7611):289-293.

10. Han X, Chen H, Huang D, et al. Mapping human pluripotent stem cell differentiation pathways using high throughput single-cell RNA-sequencing. Genome Biol. 2018;19(1):47.

11. Han X, Wang R, Zhou Y, et al. Mapping the Mouse Cell Atlas by Microwell-Seq. Cell. 2018;172(5):1091-1107 e1017. 
12. Park KS, Cha Y, Kim CH, et al. Transcription elongation factor Tcea3 regulates the pluripotent differentiation potential of mouse embryonic stem cells via the Lefty1-Nodal-Smad2 pathway. Stem Cells. 2013;31(2):282-292.

13. Spangler A, Su EY, Craft AM, Cahan P. A single cell transcriptional portrait of embryoid body differentiation and comparison to progenitors of the developing embryo. Stem Cell Res. 2018;31:201215.

14. Weinreb C, Wolock S, Klein AM. SPRING: a kinetic interface for visualizing high dimensional singlecell expression data. Bioinformatics. 2018;34(7):1246-1248.

15. Kalkan T, Smith A. Mapping the route from naive pluripotency to lineage specification. Philos Trans R Soc Lond B Biol Sci. 2014;369(1657).

16. La Manno G, Soldatov R, Zeisel A, et al. RNA velocity of single cells. Nature. 2018;560(7719):494498.

17. Butler A, Hoffman P, Smibert P, Papalexi E, Satija R. Integrating single-cell transcriptomic data across different conditions, technologies, and species. Nat Biotechnol. 2018;36(5):411-420.

18. Zhao H, Choi K. A CRISPR screen identifies genes controlling Etv2 threshold expression in murine hemangiogenic fate commitment. Nat Commun. 2017;8(1):541.

19. Sakurai H, Era T, Jakt LM, et al. In vitro modeling of paraxial and lateral mesoderm differentiation reveals early reversibility. Stem Cells. 2006;24(3):575-586.

20. Porcher C, Swat W, Rockwell K, Fujiwara Y, Alt FW, Orkin SH. The T cell leukemia oncoprotein SCL/tal-1 is essential for development of all hematopoietic lineages. Cell. 1996;86(1):47-57.

21. Liu F, Li D, Yu YY, et al. Induction of hematopoietic and endothelial cell program orchestrated by ETS transcription factor ER71/ETV2. EMBO Rep. 2015;16(5):654-669.

22. Liu F, Kang I, Park C, et al. ER71 specifies Flk-1+ hemangiogenic mesoderm by inhibiting cardiac mesoderm and Wnt signaling. Blood. 2012;119(14):3295-3305. 
23. Adams JC, Watt FM. Regulation of development and differentiation by the extracellular matrix. Development. 1993;117(4):1183-1198.

24. Mahlapuu M, Ormestad M, Enerback S, Carlsson P. The forkhead transcription factor Foxf1 is required for differentiation of extra-embryonic and lateral plate mesoderm. Development. 2001;128(2):155-166.

25. Morikawa Y, Cserjesi P. Extra-embryonic vasculature development is regulated by the transcription factor HAND1. Development. 2004;131(9):2195-2204.

26. Cheung C, Bernardo AS, Trotter MW, Pedersen RA, Sinha S. Generation of human vascular smooth muscle subtypes provides insight into embryological origin-dependent disease susceptibility. Nat Biotechnol. 2012;30(2):165-173.

27. Wareing S, Mazan A, Pearson S, Gottgens B, Lacaud G, Kouskoff V. The Flk1-Cre-mediated deletion of ETV2 defines its narrow temporal requirement during embryonic hematopoietic development. Stem Cells. 2012;30(7):1521-1531.

28. Trapnell C, Cacchiarelli D, Grimsby J, et al. The dynamics and regulators of cell fate decisions are revealed by pseudotemporal ordering of single cells. Nat Biotechnol. 2014;32(4):381-386.

29. Huynh-Thu VA, Irrthum A, Wehenkel L, Geurts P. Inferring regulatory networks from expression data using tree-based methods. PLoS One. 2010;5(9).

30. Peng G, Suo S, Chen J, et al. Spatial Transcriptome for the Molecular Annotation of Lineage Fates and Cell Identity in Mid-gastrula Mouse Embryo. Dev Cell. 2016;36(6):681-697.

31. Tripathi S, Pohl MO, Zhou Y, et al. Meta- and Orthogonal Integration of Influenza "OMICs" Data Defines a Role for UBR4 in Virus Budding. Cell Host Microbe. 2015;18(6):723-735. 